Historic, archived document

Do not assume content reflects current scientific knowledge, policies, or practices. 



\section{INSECTS ASSOCIATED WITH WILD PLANTS IN EUROPE AND THE MIDDLE EAST BIOLOGICAL CONTROL OF WEEDS SURVEYS}

UNITED STATES
DEPARTMENT OF
AGRICULTURE
MISCELLANEOUS

PUBLICATION

NUMBER 1382
PREPARED BY

SCIENCE AND

EDUCATION

ADMINISTRATION 


\begin{abstract}
Pemberton, Robert W., and Edna M. Hoover. Insects associated with wild plants in Europe and the Middle East. Biological control of weeds surveys. U.S. Department of Agriculture Miscellaneous Publication No. 1382, 33 p.
\end{abstract}

This publication lists 351 species of European insects that were collected or reared from 71 weeds of European origin. The insects speciesspecimens of which are in the USDA's Biological Control of Weeds Laboratory-Europe insect collection in Rome, Italy-are listed taxonomically and by host plant. The host plant listing includes data on rearing, feeding, and other insect-plant associations.
KEYWORDS: Biological control of weeds, insect feeding, insect rearing, host plants, weeds, Artium, Boreava, Carlina, Carthamus, Centaurea, Cirsium, Cynara, Cytisus, Echinops, Euphorbia, Galactites, Genista, Hypericum, Linaria, Onopordum, Quercus, Salsola, Salvia, Scrophularia, Senecio, Silybum, Tamarix, Tribulus.

\title{
ACKNOWLEDGMENTS
}

We would like to express our thanks to Lloyd A. Andres, Paul H. Dunn, Kenneth E. Frick, B. David Perkins (all associated at one time with the Rome laboratory), and Antonio Rizza (currently in the Rome laboratory), who allowed us to compile and publish data that they collected. We also wish to thank Gary Buckingham (former research leader at the laboratory and currently at the USDA's Biological Pest Control Research Unit, Gainesville, Fla.) whose concern for the collection and the information it contained brought the senior author to Rome to conduct the study that has resulted in this publication.

\section{CONTENTS}

Page

List of contributors . .............. iv

Introduction $\ldots \ldots \ldots \ldots \ldots \ldots \ldots$

Table 1.-Taxonomic list of insects and associated weedy plants
Table 2.-Selected weedy plants and associated insects, extent of their association, and country of origin ........ 15

Index of insect and plant names .........28 


\section{LIST OF CONTRIBUTORS}

The following scientists provided the insect identifications referred to in table 1, pages 2 to 14 : Systematic Entomology Laboratory (SEL), Insect Identification and Beneficial Insect Introduction Institute, Agricultural Research, Science and Education Administration, USDA, Beltsville Md.:

1. Anderson, W. H. ${ }^{1}$

2. Batra, S. W. T.

3. Burks, B. D. ${ }^{1}$

4. Capps, H. W. ${ }^{1}$

5. Ferguson, D. C.

6. Foote, R. H.

7. Gagne, R. J.

8. Gordh, G. ${ }^{2}$

9. Gordon, R. D.

10. Gurney, A. B. ${ }^{3}$
11. Herring, J. L. ${ }^{1}$

12. Hodges, R. W.

13. Kingsolver, J. M.

14. Knutson, L.

15. Kramer, J. P.

16. Marsh, P. M.

17. Menke, A. S.

18. Sabrosky, C. W.

19. Smith, D. R.

20. Spilman, T. J.
21. Steyskal, G. C. ${ }^{1}$

22. Stoetzel, M. B.

23. Thompson, F. C.

24. Todd, E. L. ${ }^{1}$

25. Vogt, G. B. ${ }^{3}$

26. Warner, R. E. ${ }^{1}$

27. White, R. E.

28. Whitehead, D. R.

29. Wirth, W. W.

Cooperating Scientists for SEL:

30. Arnett, R. H. ${ }^{4}$

31. Chapin, E. A. ${ }^{5}$
32. Connell, W. A. ${ }^{6}$

33. Muesebeck, C. F. W. ${ }^{7}$
34. Weld, L. H. ${ }^{5}$

Department of Entomology, U.S. National Museum, Smithsonian Institution, Washington, D.C.:

35. Davis, D. R.

36. Duckworth, W. D.
37. Field, W. D.

38. Flint, O. S.
39. Froeschner, R. C.

${ }^{1}$ Retired.

${ }^{2}$ Now at the University of California at Riverside.

${ }^{3}$ Retired, resident cooperating scientist with SEL.

${ }^{4}$ Siena College, Loudonville, N.Y.

${ }^{5}$ Deceased.

${ }^{6}$ Cooperating scientist, Beneficial Insect Research Laboratory, Newark, Del.

${ }^{7}$ Resident cooperating scientist, SEL, and research associate, U.S. National Museum, Washington D.C. 


\title{
INSECTS ASSOCIATED WITH WILD PLANTS IN EUROPE AND THE MIDDLE EAST
}

\section{Biological Control of Weeds Surveys}

\author{
Compiled by Robert W. Pemberton, collaborating entomologist, and \\ EDNA M. HOOVER, entomologist (retired)
}

\section{INTROḌUCTION}

The Biological Control of Weeds Laboratory-Europe of the United States Department of Agriculture (USDA) is located in Rome, Italy. The primary function of this facility, which was started in 1959 by Lloyd A. Andres and Antonio Rizza, is the discovery and study of natural enemies associated with weeds native to Europe and the Middle East, which are problem exotics in North America.

This publication lists the insects that have been collected and reared from some of these exotic weeds from 1959 to 1976 . The list contains only the insect species represented in the laboratory collection and thereby omits many species that were collected and reared but for various reasons are no longer in the Rome collection.

The plant taxa included in this publication consist of 23 genera and 71 identified species; 351 insect species, representing 91 families, are listed as collected or reared from these plants. Although the majority of the collections were made in Italy, material was also obtained in Austria, Bulgaria, Egypt, England, France, Germany, Greece, Iran, Israel, Spain, Switzerland, Turkey, and Yugoslavia.

The insects are listed in two tables, one tax-

${ }^{1}$ Respectively, Division of Biological Control, Department of Entomology, University of California, Berkeley, Calif. 94706; and Insect Identification and Beneficial Insect Introduction Institute, Beltsville Agricultural Research Center, Beltsville, Md. 20705. onomic (table 1, p. 2) and the other a faunal listing under the host plants (table 2, p. 15).

The large number of rearings and feeding observations listed in the host plant table is significant. Of the 351 insect species included in this work, more than half were reared; 260 are herbiverous and more than two-thirds of these were reared. In all, 317 rearing records are listed; in this context, we use rearing record to mean a unique combination of insect and host. For example, the Tephritid Urophora quadrifasciata (Meigen) was reared from Centaurea jaceae $\mathrm{L}$. and C. maculosa Lam., thus yielding two records. In addition, when the same insect species is reared in two different countries, two records are counted, even if the same host is involved.

The information contained in these tables was taken from specimen labels and laboratory records. The inclusion of precise locality and collection dates for all of the species would have made this work too lengthy. This type of information can be obtained by writing the Location Leader, Biological Control of Weeds LaboratoryEurope, c/o American Embassy, Agriculture, New York, N.Y., APO 09794.

The senior author served principally as the organizer and compiler of the data. The junior author worked with scientists in verifying the accuracy of the scientific names and in determining, from records, the persons responsible for the identifications.

The scientists of the Systematic Entomology 
Laboratory, USDA; the Department of Entomology, Smithsonian Institution; and collaborators of both organizations are contributors to this work and had the task of identifying the material and reviewing and correcting the scientific names.

The following scientists conducted the surveys that serve as a basis of this publication: Lloyd A. Andres and Paul H. Dunn, USDA Biological Control of Weeds Laboratory, Albany, Calif.; Kenneth E. Frick, USDA Delta States Agricultural Center, Stoneville, Miss.; B. David Perkins,
USDA Animal and Plant Health Inspection Service, Yaounde, Cameroon; and Antonio Rizza, USDA Biological Control of Weeds Laboratory-Europe, Rome, Italy.

This publication is intended to be a source book for biological control of weeds workers who have the task of trying to control the numerous European weeds that infest the United States. It should also be of interest to insect ecologists, plant ecologists, and insect taxonomist because of the large number of insect-plant associations it contains.

Table 1. - Taxonomic list of insects and associated weedy plants

Insect $^{1}$

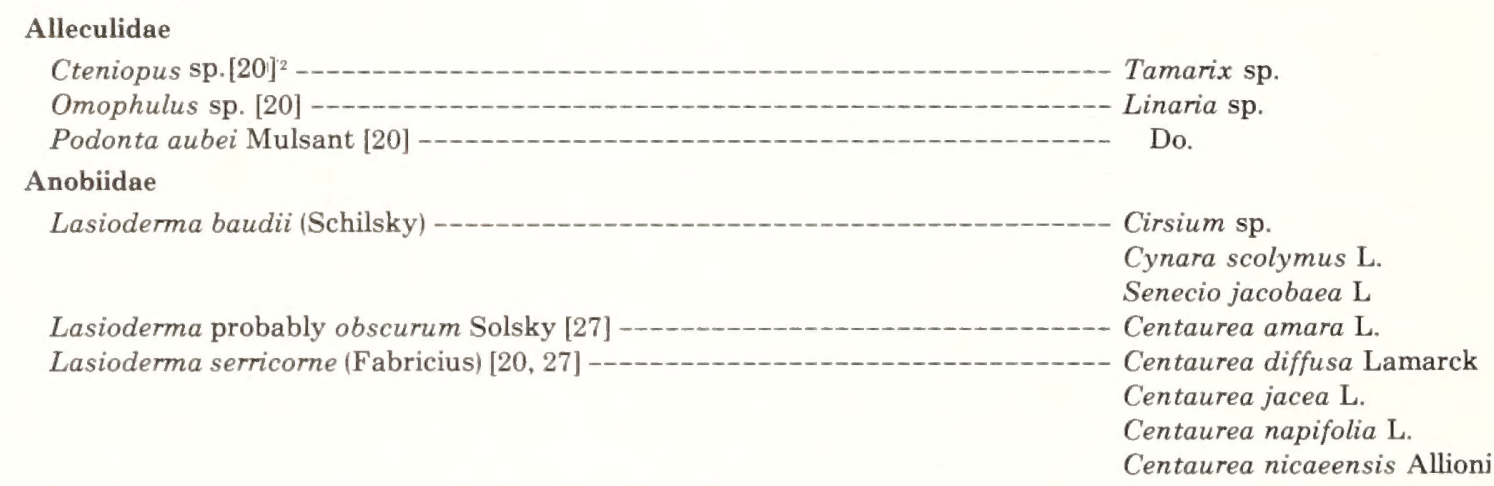

Anthicidae

Anthicus sp. [20]

Linaria sp.

Bruchidae

Bruchidius near bimaculatus (Olivier) [13]

Tamarix sp.

Bruchidius near imbricornis (Panzer) [13]

Do.

Bruchidius nudus (Allard) [13] -

Do.

Bruchidius quinqueguttatus (Olivier) complex

Centaurea repens $\mathrm{L}$.

Bruchidius seminarius (L.) [13]

Cytisus scoparius (L.) Link

Bruchidius probably unicolor (Olivier)

Linaria vulgaris Miller

Bruchidius sp. [13]

Cirsium arvense (L.) Scopoli

Bruchus brachialis Fabricius [13]

Linaria grandiflora Desfontaines

Quercus pubescens Willdenow

Bruchus dentipes Baudi [13]

Linaria genistifolia (L.) Miller

Spermophagus sericeus (Geoffroy) [13]

Centaurea repens $\mathrm{L}$.

Scrophularia canina L.

Buprestidae

Cytisus scoparius (L.) Link

Agrilus biguttatus (Fabricius) [13, 25]

Tamarix sp.

Cantharidae

Cantharis sp. [20]

Linaria dalmatica (L.) Miller

Podabrus sp. [9]

Cerambycidae

Agapanthia cardui (L.) $[20,25]$

Galactites tomentosa Moench

Agapanthia dahli (Richter) [20, 25]

Cirsium sp.

See footnotes at end of table.

Galactites tomentosa Moench 
Table 1. - Taxonomic list of insects and associated weedy plants-Continued

Insect ${ }^{1}$ Associated plant

\section{COLEOPTERA-Continued}

Cerambycidae-Continued

Agapanthia probably dahli (Richter) [20]

Cartallum ebulinum (L.) [20, 25]

Senecio aquaticus Hill

Parmena sp. [20]

Boreava sp.

Phytoecia pustulata (Schrank) [20, 25]

Centaurea cineraria $\mathrm{L}$.

Phytoecia sp. $[20,25]$

Salvia verbenaca $\mathrm{L}$.

Linaria fastigiata Chavannes

Linaria genistifolia (L.) Miller

Linaria kurdica Boissier \& Hohenacker

\section{Chrysomelidae}

Altica carduorum (Guérin-Méneville) [27]

Cirsium arvense (L.) Scopoli

Cirsium vulgare (Savi) Tenore

Cassida near deflorata Suffrian [25, 27]

Silybum marianum (L.) Gaertner

Cassida probably vibex L. [25, 27]

Cirsium arvense (L.) Scopoli

Chaetocnema probably conducta Motschulsky [27]

Silybum marianum (L.) Gaertner

Chrysolina grossa (Fabricius) [27]

Senecio aquaticus Hill

Chrysolina gypsophilae Küster [27]

Salvia sp.

Chrysolina hyperici Forster [27]

Linaria dalmatica (L.) Miller

Chrysolina variolosa Pethena [27]

Hypericum sp.

Chrysolina sp. [27]

Linaria sp.

Linaria vuigaris Miller

Linaria sp.

Chrysomela sp. or near [27]

Cytisus scoparius (L.) Link

Clytra atraphaxidis Pallas or near [27]

Centaurea repens $\mathrm{L}$.

Clytra laeviuscula Ratzeburg or near [27]

Linaria sp.

Probably Coptocephala sp. [27]

Centaurea repens $\mathrm{L}$.

Crepidodera ferruginea (Scopoli) or near [27]

Onopordum tauricum Willdenow

Cryptocephalus hypochoeridis (L.) [27]

Onopordum sp.

Cryptocephalus populi Suffrian or near [27]

Salsola sp.

Diorhabda sp. [27]

Tamarix sp.

Gastroidea polygoni (L.) [27]

Cynara scolymus L.

Senecio aquaticus Hill

Silybum marianum (L.) Gaertner

Lema cyanella L. [27]

Longitarsus dorsalis (Fabricius) [27]

Cirsium arvense (L.) Scopoli

Longitarsus ganglbaueri Heikertinger [27]

Senecio aquaticus Hill

Longitarsus probably ganglbaueri Heikertinger [27]

Do.

Do.

Senecio jacobaea L.

Longitarsus jacobaea Waterhouse [27]

Senecio aquaticus Hill

Longitarsus probably jacobaea Waterhouse [27]

Do.

Senecio jacobaea L.

Longitarsus near tabidus (Fabricius) [27]

Senecio aquaticus Hill

Longitarsus sp. [27]

Senecio jacobaea $\mathrm{L}$.

Oulema melanopus (L.) [27]

Senecio aquaticus Hill

Senecio jacobaea L.

Pachybrachys sp. [27]

Boreava sp.

Phyllotreta sp. [27]

Boreava sp.

Linaria sp.

Phytodecta olivacea Forster

Cytisus scoparius (L.) Link

Sermylassa halensis (L.) [27]

Senecio jacobaea $\mathrm{L}$.

Sphaeroderma rubidum (Graells) [25]

Cirsium arvense (L.) Scopoli

Cynara scolymus L.

Sphaeroderma testaceum L. [25, 27]

Cirsium arvense (L.) Scopoli

\section{Coccinellidae}

Adonia variegata (Goeze) $[9,31]$

Linaria sp.

See footnotes at end of table. 
Table 1. - Taxonomic list of insects and associated weedy plants-Continued

Insect ${ }^{1}$ Associated plant

COLEOPTERA-Continued

Coccinellidae-Continued

Coccinella quinquepunctata L. [31] -

Coccinella septempunctata L. [9,31]

Do.

Do.

Tamarix sp.

Rhyzobius litura (Fabricius) [31]

Centaurea napifolia $\mathrm{L}$.

Scymnus (s.str.) sp. [9] -_._._...- Linaria sp.

Scymnus sp. [31] -

Linaria sp.

Subcoccinella vigintiquattuorpunctata $(\mathrm{L}).[9,31]$

Cynara scolymus L.

Linaria sp.

Curculionidae

Acalles diocletianus (Germar)

Cynara scolymus L.

Acalles dromedarius Boheman

Centaurea napifolia $\mathbf{L}$.

Cynara scolymus L.

Acalles sp. [26]

Centaurea napifolia $\mathrm{L}$.

Centaurea nicaeensis Allioni

Centaurea sp.

Alophus nictitans Boheman [28]

Senecio aquaticus Hill.

Apion near compactum Desbrochers [26]

Cytisus sp.

Apion detritum Rey [26]

- Centaurea amara L.

Cirsium arvense (L.) Scopoli

Silybum marianum (L.) Gaertner

Apion near formaneki Wagner [26]

Genista sagittalis L.

Apion fuscirostre (Fabricius) [26]

Cytisus scoparius (L.) Link

Apion onopordi Kirby [26, 28]

Centaurea maculosa Lamarck

Onopordum tauricum Willdenow

Apion sp. [26, 28]

Centaurea nicaeensis Allioni

Cirsium vulgare (Savi) Tenore

Genista sp.

Linaria sp.

Tamarix sp.

Bangasternus sp. [26]

Centaurea jacea L.

Ceutorhynchidius horridus (Panzer) [26, 28]

Galactites tomentosa Moench

Ceutorhynchus litura (Fabricius) [26]

Cirsium arvense (L.) Scopoli

Ceutorhynchus trimaculatus (Fabricius) [26]

Galactites tomentosa Moench

Chlorophanus excisus (Fabricius) [28]

Tamarix sp.

Cleonus morbillosus (Fabricius) [26] -_-_-_-_-_-_._-_ium vulgare (Savi) Tenore

Cleonus piger (Scopoli) [26, 28]

Arctium minus Bernhardi

Cirsium arvense (L.) Scopoli

Cirsium vulgare (Savi) Tenore

Onopordum tauricum Willdenow

Coniatus sp. [28]

Tamarix sp.

Gymnetron antirrhini (Paykull) [26]

Linaria vulgaris Miller

Gymnetron linariae (Panzer) [26]

Do.

Gymnetron netum (Germar) [26]

Linaria heterophylla Desfontaines

Linaria vulgaris Miller

Gymnetron sp. [26, 28]

Centaurea nicaeensis Allioni

Linaria dalmatica (L.) Miller

Linaria fastigiata Chavannes

Linaria genistifolia (L.) Miller

Linaria lineolata Boissier

Linaria repens (L.) Miller

Linaria vulgaris Miller

Hypera postica (Gyllenhal) [26, 28]

Centaurea napifolia $\mathrm{L}$.

See footnotes at end of table. 
Table 1. - Taxonomic list of insects and associated weedy plants-Continued

Insect $^{1}$ Associated plant

COLEOPTERA-Continued

Curculionidae-Continued

Larinus australis Capiomont [26]

Centaurea phrygia $\mathrm{L}$.

Onopordum tauricum Willdenow

Larinus probably australis Capiomont [26]

Centaurea sp.

Larinus cynarae (Fabricius) $[26,28]$

Onopordum tauricum Willdenow

Onopordum sp.

Silybum marianum (L.) Gaertner

Larinus flavescens Germar [26]

Larinus jaceae (Fabricius) [26, 28]

Carthamus lanatus $\mathrm{L}$

Centaurea nicaeensis Allioni

Larinus latus (Herbst)

Carthamus lanatus L.

Larinus near leuzeae Fabre [26] -

- Onopordum sp.

Larinus longirostris Gyllenhal [26]

- Centaurea scabiosa L.

- Centaurea calcitrapa L.

Centaurea napifolia $\mathrm{L}$.

Centaurea nicaeensis Allioni

Larinus planus (Fabricius) [26]

Centaurea amara $\mathrm{L}$.

Centaurea jacea $\mathrm{L}$.

Cirsium arvense (L.) Scopoli

Cirsium vulgare (Savi) Tenore

Larinus rusticanus Gyllenhal [26, 28]

Carthamus lanatus L.

Larinus scolymi (Olivier) [26, 28]

Cynara scolymus L.

Larinus sturnus (Schaller) [26, 28]

- Centaurea jacea L.

Cirsium vulgare (Savi) Tenore

Larinus turbinatus Gyllenhal [26]

Cirsium arvense (L.) Scopoli

Larinus ursus (Fabricius) [26, 28]

Cirsium vulgare (Savi) Tenore

Carlina corymbosa $\mathrm{L}$.

Carlina lanata L.

Carlina vulgaris $\mathrm{L}$.

Lixus algirus (L.) [28]

Cirsium vulgare (Savi) Tenore

Silybum marianum (L.) Gaertner

Lixus cardui Olivier [28]

Onopordum acanthium L.

Onopordum sp.

Lixus elongatus (Goeze) [26, 25]

Cirsium vulgare (Savi) Tenore

Lixus punctiventris Boheman

- Senecio aquaticus Hill

Senecio jacobaea L.

Mecinus janthinus (Germar) [26]

Linaria vulgaris Miller

Mecinus sp. [26]

Linaria sp.

Microlarinus lareynii (Jacq. du Val) [26]

Tribulus terrestris $\mathrm{L}$.

Microlarinus lypriformis (Wollaston) [26]

Do.

Otiorhynchus ovatus (L.) [26]

Centaurea mauclosa Lamarck

Otiorhynchus rugostriatus (Goeze) [26]

Senecio aquaticus Hill

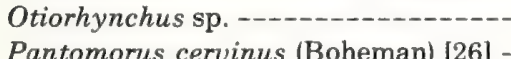

Cirsium arvense (L.) Scopoli

Pantomorus cervinus (Boheman) [26]

Senecio jacobaea $\mathbf{L}$.

Phrydiuchus spilmani Warner [26]

Salvia argentea $\mathrm{L}$.

Phrydiuchus tau Warner [26]

Salvia aethiopis L.

Phyllobius sp.

Cirsium vulgare (Savi) Tenore

Rhinocyllus conicus (Froelich) [26, 28]

- Centaurea jacea L.

Centaurea sp.

Silybum marianum (L.) Gaertner

Sibinia attalica (Gyllenhal)

Centaurea amara L.

Centaurea caerulescens Willdenow

Sitona gressorius (Fabricius) [28]

Cirsium vulgare (Savi) Tenore

Sitona lineatus (L.) [28]

Senecio jacobaea L.

Sitona puncticollis Stephens

Senecio aquaticus Hill

See footnotes at end of table. 
Table 1. - Taxonomic list of insects and associated weedy plants-Continued

Insect ${ }^{1}$ Associated plant

COLEOPTERA-Continued

Curculionidae-Continued

Sitona sp. $[26,28]$

Centaurea jacea $\mathrm{L}$.

Cirsium arvense (L.) Scopoli

Cirsium vulgare (Savi) Tenore

Cynara scolymus L.

Echinops sphaerocephalus L.

Sitophilus sp. [28]

Onopordum tauricum Willdenow

Strophomorphus porcellus (Schonherr) [26]

Cynara scolymus L.

Tychius quinquepunctatus (L.)

- Echinops sphaerocephalus L.

Elateridae

Cardiophorus near nigerrimus Erichson [20]

Cirsium arvense (L.) Scopoli

Lathridiidae

Corticaria sp. [13]

Linaria sp.

Meloidae

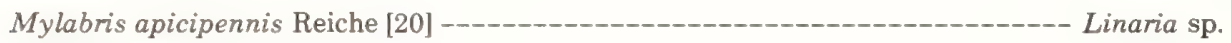

Mylabris sp. [20] -

Melyridae

Aplocnemus sp. [13] -

Attalus sp. $[13,25]--1$,

Centaurea repens $\mathrm{L}$.

Linaria vulgaris Miller

Colotes sp. [13]

Linaria sp.

Danacaea sp. $[13,25]$

Centaurea calcitrapa L.

Probably Danacaea sp. [13]

Linaria vulgaris Miller

Linaria sp.

Divales bipustulata Fabricius [13]

Centaurea caerulescens Willdenow

Divales sp. [13] Do.

Julistus sp. [13]

Linaria heterophylla Desfontaines

Malachius aeneus L. [13, 25]

Malachius sp. [13, 25]

Tamarix sp.

Psilothrix sp. $[13,25]-\ldots$

\section{Mordellidae}

Anaspis sp. [20]

Mordellistena sp. [20]

Nitidulidae

Brachypterolus pulicarius (L.) [32]

Brachypterolus sp. [32]

Epuraea sp. [32]

Meligethes incanus Sturm [32]

Meligethes sp. [32]

\section{Oedemeridae}

Oedemera podagrariae (L.) [30]

See footnotes at end of table.
Cytisus scoparius (L.) Link

- Centaurea nicaeensis Allioni

Linaria dalmatica (L.) Miller Linaria fastigiata Chavannes Linaria genistifolia (L.) Miller Linaria grandiflora Desfontaines Linaria heterophylla Desfontaines Linaria kurdica Boissier \& Hohenacker Linaria lineolata Boissier Linaria vulgaris Miller Linaria sp.

Linaria genistifolia (L.) Miller Linaria vulgaris Miller Cirsium arvense (L.) Scopoli Centaurea maculosa Lamarck Salvia aethiops L.

Cirsium arvense (L.) Scopoli

Onopordum sp. 
Table 1. - Taxonomic list of insects and associated weedy plants-Continued

Insect $^{1}$

Associated plant

COLEOPTERA-Continued

Orthoperidae

Arthrolips sp. [9]

Tribulus terrestris $\mathrm{L}$.

Phalacridae

Olibrus corticalis (Panzer) [13] _.

Ptinidae

Ptinus villiger (Reitter) [20]

Centaurea cineraria $\mathbf{L}$.

\section{Scarabaeidae}

Glaphyrus sp. [9] -

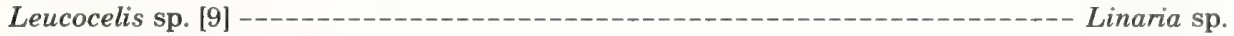

Valgus hemipterus L. [9] --_-

Scolytidae

Phloeotribus rhododactylus (Marsham) [1] _-_._-_._- Cytisus scoparius (L.) Link

\section{DIPTERA}

Agromyzidae

Liriomyza latigenis (Hendel)

Senecio jacobaea L.

Liriomyza strigata (Meigen)

Senecio aquaticus Hill

Melanagromyza aeneoventris (Fallén) -

Senecio jacobaea $\mathbf{L}$.

Melanagromyza dettmeri Hering

Senecio aquaticus Hill

Melanagromyza eupatorii Spencer

Senecio jacobaea L.

Melanagromyza near tripolii Spencer [21]

Senecio aquaticus Hill

Ophiomyia senecionina Hering

Senecio jacobaea L.

Phytomyza probably horticola Goureau [21]

Centaurea calcitrapa $\mathrm{L}$.

Linaria sp.

Anthomyiidae

Hylemya brassicae (Wiedemann) [21]

Senecio jacobaea L.

Hylemya cinerella (Fallén) [21]

Do.

Hylemya coarctata (Fallén [21] --

Hylemya platura (Meigen) [21]

Hylemya pullula (Zetterstedt) [21] -

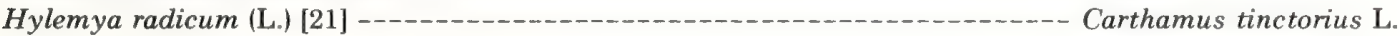

Senecio jacobaea L.

Hylemya seneciella (Meade) [21]

Do.

Hylemya sp. [21]

Tamarix sp.

Anthomyzidae

Anthomyza gracilis Fallén [18]

Centaurea jacea L.

Calliphoridae

Bufolucilia silvarum (Meigen)

Senecio jacobaea L.

Chamaemyiidae

Leucopis sp. [18, 21]

Centaurea nicaeensis Allioni

Linaria sp.

\section{Chloropidae}

Oscinella frit (L.) [18]

Centaurea jacea $\mathrm{L}$.

Oscinella sp. [18]

Linaria genistifolia (L.) Miller

Polydodaspis ruficornis (Macquart) [18]

Centaurea jacea L.

Thaumatomyia sulcifrons (Becker) [18]

Centaurea repens $\mathrm{L}$.

Drosophilidae

Drosophila funebris (Fabricius) [29]

Cynara scolymus L.

Empididae

Microphorus sp. [14]

Tamarix sp.

Platypalpus sp. [14, 21]

Linaria grandiflora Desfontaines

Tamarix sp.

See footnotes at end of table. 
Table 1. - Taxonomic list of insects and associated weedy plants-Continued

Insect $^{1}$ Associated plant

DIPTERA-Continued

Lauxaniidae

Minettia plumicornis (Fallén) [21]

Lonchopteridae

Lonchoptera furcata (Fallén) [29]

Tamarix sp.

Milichiidae

Desmometopa near m-nigrum (Zetterstedt) [18]

Centaurea nicaeensis Allioni

Muscidae

Fannia sp. [21]

Orthellia caesarion (Meigen) [7]

Tamarix sp

Otitidae

Timia xanthaspis (Loew)

Centaurea repens $\mathrm{L}$.

Phoridae

Phora sp. [29]

Cirsium arvense (L.) Scopoli

Sarcophagidae

Sarcophaga balanina Pandellé [7]

Tribulus terrestris L.

Sarcophaga sp. [7]

Centaurea jacea L.

Sphaeroceridae

Leptocera sp. [18, 21]

Linaria genistifolia (L.) Miller

Stratiomyidae

Nemotelus sp. [29]

Linaria sp.

Syrphidae

Eumerus strigatus Fallén

Centaurea repens $\mathrm{L}$.

Eumerus sp.

Senecio jacobaea L.

Paragus sp. [23]

Linaria sp.

Sphaerophoria rueppelli Wiedemann [23]

Senecio jacobae $\mathbf{L}$.

Tachinidae

Voria ruralis (Fállen) [18]

Cynara cardunculus L.

Tephritidae

Acanthiophilus helianthi (Rossi) $[6,21]$

- Carthamus lanatus L.

Centaurea amara $\mathrm{L}$.

Centaurea caerulescens Willdenow

Centaurea calcitrapa L.

Centaurea jacae L.

Centaurea maculosa Lamarck

Centaurea napifolia $\mathrm{L}$.

Centaurea nicaeensis Allioni

Centaurea paniculata L.

Centaurea repens $\mathrm{L}$.

Cynara scolymus L.

Onopordum sp.

Chaetorellia sp. [6, 21]

Carthamus tinctorius L.

Centaurea amara L.

Centaurea jacae L.

Chaetostomella onotrophes (Loew) [6]

Do.

Centaurea nigra L.

Centaurea phrygia L.

Centaurea scabiosa L.

Centaurea sp.

Cirsium arvense (L.) Scopoli

Orellia colon (Meigen) [21]

Centaurea scabiosa L.

Orellia near colon (Meigen) [6]

Carthamus lanatus L.

Orellia falcata (Scopoli) [6]

Centaurea jacae L.

See footnotes at end of table. 
Table 1. - Taxonomic list of insects and associated weedy plants-Continued

Insect $^{1}$ Associated plant

DIPTERA - Continued

Tephritidae-Continued

Orellia lappae (Cederhjelm) [6, 21]

Orellia punctata (Schrank) [6]

Onopordum tauricum Willdenow

Onopordum sp.

Orellia near punctata (Schrank) [6]

Centaurea schaerocephala $\mathrm{L}$.

Orellia sp. [6]

Centaurea nicaeensis Allioni

Oxyna nebulosa (Wiedemann) [21]

- Centaurea scabiosa L.

Paroxyna probably tessellata (Loew)

Senecio jacobaea L.

Sphenella marginata (Fallén) [21]

Do.

Tephritis postica (Loew) [6]

Do.

Onopordum tauricum Willdenow Onopordum sp.

Terellia fuscicornis (Loew)

Cynara scolymus L.

Terellia longicauda (Meigen) [6]

Cirsium vulgare (Savi) Tenore

Terellia serratulae (L.) $[6,21]$

Centaurea sp.

Terellia virens (Loew) [21]

Centaurea maculosa Lamarck

Terellia near virens (Lowe) [6]

Onopordum sp.

Trupanea stellata (Fuessly) [21]

Senecio jacobaea $\mathrm{L}$.

Senecio jacobaea L.?

Urophora affinis (Frauenfeld) [6, 21] -_-_-_entaurea maculosa Lamarck

Urophora cuspidata (Meigen) [6] --_-_entaurea scabiosa L.

Urophora jaceana (Hering) [6] --_-_- Centaurea nigra L.

Urophora quadrifasciata (Meigen) [6, 21] -...-...- Centaurea jacea L.

Centaurea maculosa Lamarck

Urophora probably quadrifasciata (Meigen) [6, 21]

Centaurea amara $\mathrm{L}$.

Centaurea calcitrapa L.

Centaurea diffusa Lamarck

Centaurea jacea $\mathrm{L}$.

Centaurea maculosa Lamarck

Centaurea nicaeensis Allioni

Centaurea scabiosa $\mathrm{L}$.

Urophora stylata (Fabricius) $[6,21]$

Cirsium arvense (L.) Scopoli

Cirsium vulgare (Savi) Tenore

Cirsium sp.

Trixoscelidae

Trixoscelis frontalis (Fallén) [21]

Trixoscelis obscurella (Fallén) [21]

Tamarix sp.

Do.

\section{HEMIPTERA}

Anthocoridae

Anthocoris nemorum (L.) [11]

Linaria sp.

\section{Berytidae}

Berytinus sp. [11]

Scrophularia canina L.

\section{Coreidae}

Enoplops discigera (Kolenati) [11]

Linaria sp.

Mesocerus marginatus (L.) ? [11]

Onopordum sp.

Scrophularia canina L.

Strobilotoma typhaecornis (Fabricius) [11]

Do.

Lygaeidae

Cymus glandicolor Hahn [11]

Linaria vulgaris Miller

Geocoris megacephalus siculus (Fieber) [11]

Salsola sp.

Heterogaster urticae (Fabricius) [11]

Scrophularia canina L.

Lygaeus equestris (L.) [11]

Linaria sp.

Sphragisticus nebulosus (Fallén) [11]

Salvia virgata Jacquin

Spilostethus saxatilis (Scopoli) [11]

Linaria sp.

\section{Miridae}

Adelphocoris lineolatus (Goeze) [39]

Do.

See footnotes at end of table. 
Table 1. - Taxonomic list of insects and associated weedy plants-Continued

Insect $^{1}$ Associated plant

HEMIPTERA-Continued

Miridae-Continued

Adelphocoris sp. [39] .

Alloeonotus fulvipes (Scopoli) [39]

Do.

Brachycoleus decolor Reuter [11, 39]

Do.

Deraeocoris schach (Fabricius) [11]

Do.

Lygus prantensis (L.) [39]

Onopordum tauricum Willdenow

Lygus rugulipennis Poppius [39]

Linaria sp.

Polymerus sp. [39]

Do.

Nabidae

Nabis viridulus Spinola [39]

Do.

\section{Pentatomidae}

Carpocoris lunulatus (Goeze) [39]

Carpocoris mediterraneus Taminini [11, 39]

Tamarix sp.

Carpocoris pudicus (Poda) [39]

Linaria sp.

Do.

Silybum marianum (L.) Gaertner

Dolycoris baccarum (L.) $[11,39]$ Linaria sp.

Do.

Onopordum sp.

Tamarix sp.

Eurydema ornatum (L.) [11, 39]

Linaria dalmatica (L.) Miller

Eurygaster maura (L.) [39]

Linaria sp.

Eysarcoris inconspicuus (Herrich-Schäffer) [11]

Do.

Holcostethus strictus (Fabricius) [11]

Linaria vulgaris Miller

Holcostethus sp. [11]

Centaurea repens $\mathrm{L}$.

Nezara viridula (L.) [11, 39]

Linaria dalmatica (L.) Miller

Palomena prasina L.) [11]

Onopordum sp.

Phymatidae

Phymata crassipes (Fabricius) ? [11]

Scrophularia canina L.

Podopidae

Ancyrosoma leucogrammum (Gmelin) [11]

Graphosoma lineata (L.) [11]

Reduviidae

Rhinocoris iracundus Poda [39]

Linaria sp.

Rhopalidae

Corizus hyoscyami L. [11]

Do.

Rhopalus sp. [11] -

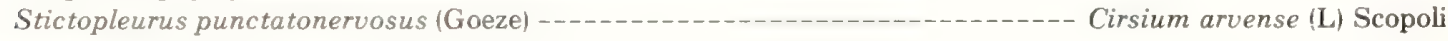

Tingidae

Dictyla echii (Schrank) [39]

Linaria sp.

\section{HOMOPTERA}

\section{Cercopidae}

Cercopis vulnerata Rossi [15]

Philaenus spumarius (L.) [15, 39]

Onopordum tauricum Willdenow

Cirsium arvense (L.) Scopoli

Onopordum tauricum Willdenow

Senecio aquaticus Hill

Cicadellidae

Circulifer opacipennis (Lethierry) [15] -- -

Deltocephalus sp. [15] -

Handianus sp. [15] -

Hephathus nanus (Herrich-Schäffer) [15] -.........-...-. Centaurea calcitrapa L.

Cicadidae

Cicadatra near hyalina (Fabricius) [39]

Linaria sp.

See footnotes at end of table. 
Table 1. - Taxonomic list of insects and associated weedy plants-Continued

\section{Insect $^{1}$} Associated plant

\section{HOMOPTERA-Continued}

\section{Cixiidae}

Hyalesthes obsoletus Signoret [15]

Oliarus barajus Dlabola [15]

Centaurea repens $\mathrm{L}$.

Issidae

Hysteropterum grylloides (Fabricius) [15]

Do.

\section{Tettigometridae}

Tettigometra griseola Fieber [15]

Centaurea calcitrapa $\mathrm{L}$.

Tettigometra sp. [15]

Centaurea repens $\mathrm{L}$.

HYMENOPTERA

Anthophoridae

Ceratina sp. [2]

Tamarix sp.

\section{Braconidae}

Apanteles sp. [16, 33]

Senecio jacobaea L.

Biosteres carbonaris (Nees) [16]

Cynara scolymus $\mathbf{L}$.

Bracon near osculator Nees [16]

Linaria sp.

Bracon sp. [16]

- Centaurea maculosa Lamarck

Cynara scolymus L.

Linaria vulgaris Miller

Senecio aquaticus Hill

Chelonus sp. [16]

Senecio jacobaea L.

Dacnusa leptogaster Haliday

Linaria vulgaris Miller

Microplitis possibly scrophulariae Szelenyi [16]

Do.

Microplitis possibly variipes Ruthe [16]

Linaria sp.

Opius sp. [16]

Do.

Orgilus sp. [16]

Senecio jacobaea $\mathrm{L}$.

Cynipidae

Diglyphosema sp. [17]

Do.

Eutrias sp. [17]

Do.

Isocolus scabiosae (Giraud) [34]

Do.

- Centaurea amara L.

Centaurea caerulescens Willdenow

Centaurea cineraria $\mathrm{L}$.

Centaurea napifolia $\mathrm{L}$.

Centaurea phrygia $\mathrm{L}$.

Centaurea scabiosa $\mathrm{L}$.

\section{Diapriidae}

Psilus sp. [16]

Senecio jacobaea L.

\section{Eulophidae}

Entedon sp. [3, 8]

Linaria sp.

\section{Eupelmidae}

Eupelmus sp. [3]

Do.

\section{Eurytomidae}

Eudecatoma biguttata Swederus or E. submutica (Thomson) [3]

Centaurea caerulescens Willdenow Centaurea jacea $\mathrm{L}$.

Centaurea napifolia L.

Centaurea nicaeensis Allioni

Eudecatoma submutica (Thomson) [3]

Centaurea caerulescens Willdenow

Centaurea jacea L.

Centaurea napifolia $\mathrm{L}$.

Centaurea nicaeensis Allioni

See footnotes at end of table. 
Table 1. - Taxonomic list of insects and associated weedy plants-Continued

Insect

Associated plant

\section{HYMENOPTERA-Continued}

Eurytomidae-Continued

Eurytoma robusta Mayr [3]

Carthamus lanatus L.

Centaurea caerulescens Willdenow

Centaurea jacea $\mathrm{L}$.

Centaurea nicaeensis Allioni

Centaurea phrygia L.

Centaurea scabiosa L.

Centaurea sp.

Cirsium arvense (L.) Scopoli

Cirsium vulgare (Savi) Tenore

Onopordum sp.

Eurytoma robusta Mayr or E. tibialis Boheman [3]

Echinops sphaerocephalus L.

Eurytoma tibialis Boheman [8]

Centaurea caerulescens Willdenow

Centaurea calcitrapa L.

Centaurea jacea L.

Centaurea maculosa Lamarck

Centaurea nicaeensis Allioni

Centaurea phrygia L.

Centaurea scabiosa L.

Cirsium arvense (L.) Scopoli

Cirsium vulgare (Savi) Tenore

Eurytoma sp. [3]

Linaria sp.

Formicidae

Camponotus lateralis (Olivier) [19]

Linaria sp.

Cataglyphis near cursor (Fonscolombe) [19] -...--

Formica near nasuta Nylander [19] -

Formica sp. [19] -_-

Tapinoma sp. [19] -..........

Mymaridae

Anaphes sp. [3]

Do.

Ormyridae

Ormyrus gratiosus (Foerster) [3]

Centaurea caerulescens Willdenow Centaurea clacitrapa $\mathrm{L}$.

Ormyrus punctiger Westwood [3]

Centaurea amara $\mathrm{L}$.

Centaurea caerulescens Willdenow

Centaurea jacea L.

Centaurea maculosa Lamarck

Centaurea nicaeensis Allioni

Cirsium arvense (L.) Scopoli

Onopordum sp.

Pteromalidae

Caenacis sp. [3]

Centaurea sp.

Spintherus sp. [3]

Linaria sp.

Scelionidae

Telenomus sp. [16] -

\section{LEPIDOPTERA}

Arctiidae

Tyria jacobaeae (L.)

Senecio jacobaea L.

Cochylidae

Cochylis probably atricapitana (Stephens)

Senecio aquaticus Hill

Senecio jacobaea L.

Cochylis near hybridella (Hübner) [35]

Cirsium arvense (L.) Scopoli

Cochylis posterana (Zeller)

Centaurea calcitrapa L.

Onopordum sp.

See footnotes at end of table. 
Table 1. - Taxonomic list of insects and associated weedy plants-Continued

Insect $^{1}$ Associated plant

\section{LEPIDOPTERA-Continued}

\section{Coleophoridae}

Coleophora sp. $[12,35]$

Centaurea nicaeensis Allioni

Salsola kali L.

\section{Cosmopterigidae}

Pyroderces argyrogrammos (Zeller) [12]

Carthamus lanatus $\mathrm{L}$.

Centaurea amara $\mathrm{L}$.

Centaurea calcitrapa L.

Centaurea sphaerocephala $\mathrm{L}$.

Cirsium arvense (L.) Scopoli

Cynara scolymus L.

Galactites tomentosa Moench

Onopordum sp.

Stagmatophora serratella (Treitschke) [12]

Silybum marianum (L.) Gaertner

Stagmetophora serratella (Treitschke)

Linaria dalmatica (L.) Miller

Linaria repens (L.) Miller

Linaria vulgaris Miller

Linaria sp.

\section{Geometridae}

Chlorissa sp.

Senecio aquaticus Hill

Ematurga atomaria (L.) [24]

Senecio jacobaea L.

Eupithecia absinthiata (Clerck) [12]

Do.

Eupithecia centaurearia (Denis \& Schiffermüller) [24]

Senecio aquaticus Hill

Senecio jacobaea L.

Eupithecia linariata (Denis \& Schiffermüller) [24]

Linaria vulgaris Miller

Eupithecia sp. [5]

Gymnoscelis pumilata (Hübner) [25]

Cirsium vulgare (Savi) Tenore

Linaria vulgaris Miller

\section{Lyonetiidae}

Leucoptera spartifoliella (Hübner)

Cytisus scoparius (L.) Link

\section{Noctuidae}

Calophasia casta (Borkhausen) [24]

Linaria dalmatica (L.) Miller

Linaria sp.

Heliothis peltiger (Denis \& Schiffermüller) [24]

Centaurea calcitrapa L.

Heliothis sp. [24]

Salvia limbata C. A. von Meyer

Peridroma saucia (Hübner) [24] -..-... Senecio squalidus L.

Phytometra sp. [24] -._-_.-- Salvia verbenaca $\mathrm{L}$.

Porphyrinia parva (Hübner) [24]

Carthamus lanatus L.

Centaurea amara $\mathbf{L}$.

Centaurea calcitrapa L.

Centaurea napifolia $\mathrm{L}$.

Porphyrinia purpurina (Denis \& Schiffermüller) [24]

Cirsium odontolepis Boissier

Prodotis stolida (Fabricius) [24]

Centaurea amara $\mathrm{L}$.

Cynara scolymus L.

\section{Nymphalidae}

Cynthia cardui (L.) [37]

Salvia palaestina Bentham

Melitaea cinxia (L.) [37] Silybum marianum (L.) Gaertner

Melitaea didyma (Esper) [37] Cynara scolymus L.

Linaria vulgaris Miller

Linaria sp.

\section{Oecorphoridae}

Depressaria sp.

Centaurea cineraria L.

Plutellidae

Plutella xylostella (L.) [12]

Linaria sp.

See footnotes at end of table. 
Table 1. - Taxonomic list of insects and associated weedy plants-Continued

Insect In $^{1}$

Associated plant

LEPIDOPTERA-Continued

Pyralidae

Gymnancyla canella (Denis \& Schiffermüller)

Salsola kali L.

Homeosoma near nebulella (Denis \& Schiffermüller) -_-_-_-_-_-_-_-_-_-_--_ Cirsium vulgare (Savi) Tenore

Homeosoma sp. [36] ----

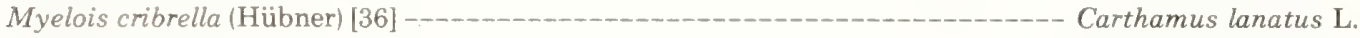

Myelois sp. [36] --

Palpita unionalis (Hübner) [4] --_--_-_-_-_-_-_-_-_-_-_-_-_---_ Linaria vulgaris Miller

Probably Phycitodes albatella (Ragonot) ----------------------------- Senecio jacobaea L.

Phycitodes carlinella Heinemann) --_-_._- Senecio aquaticus Hill

Sesiidae

Chamaesphecia empiformis (Esper) --_-_- Euphorbia esula L.

Sphingidae

Hyles euphorbiae (L.) [12] -- Euphorbia sp.

\section{Tortricidae}

Cacoecimorpha pronubana (Hübner) [12] -------------------.------ Cynara scolymus L.

Senecio ercifolius L.

Senecio nemorensis L.

Cnephasia sp. [12]

Centaurea napifolia $\mathrm{L}$.

Epiblema near pflugiana (Fabricius)

Silybum marianum (L.) Gaertner

Eucosma campoliliana (Denis \& Schiffermüller)?

Cirsium vulgare (Savi) Tenore

Exartema sp. [35]

Senecio jacobaea L.

Zygaenidae

Adscita sp. [12]
NEUROPTERA

Do.

Chrysopidae

Chrysopa near carnea Stephens [38]

Linaria sp.

\section{ORTHOPTERA}

Tettigoniidae

Tettigonia caudata (Charpentier) [10]

Do.

\footnotetext{
${ }^{1}$ Insects are listed alphabetically by order and family.

* The numbers in brackets following the scientific name refer to the numbers in the List of Contributors, p. iv., and signify the person or persons who provided the identification. Where no numbers are given, the identifier is not known.
} 
Table 2.- Selected weedy plants and associated insects, extent of their association, and country of origin

Plant and insect ${ }^{1}$ Insect-plant

relationship ${ }^{2}$

Country

Arctium minus Bernhardi:

Cleonus piger (Scopoli) [Curculionidae, CO]

Reared from root

Italy.

Boreava sp.:

Cartallum ebulinum (L.) [Cerambycidae, CO]

Sweapt from plant

Turkey.

Phyllotreta sp. [Chrysomelidae, CO]

Do.

Carlina corymbosa $\mathbf{L}$.:

Larinus ursus (Fabricius) [Curculionidae, CO]

Feeding on head

Italy.

Carlina lanata $\mathbf{L}$.:

Larinus ursus (Fabricius) [Curculionidae, CO]

Reared from head

Do.

Carlina vulgaris $\mathbf{L}$ :

Larinus ursus (Fabricius) [Curculionidae, CO]

Larinus ursus (Fabricius) [Curculionidae, CO]

Feeding on plant

Do.

Carthamus lanatus $\mathbf{L}$ :

Acanthiophilus helianthi (Rossi) [Tephritidae, DI]

Reared from head

Eurytoma robusta Mayr [Eurytomidae, HY]

Larinus flavescens Germar [Curculionidae, CO]

Larinus flavescens Germar [Curculionidae, CO]

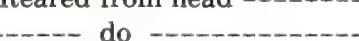

Larinus jaceae (Fabricius) [Curculionidae, CO]

Feeding on head

Reared from head

Larinus jaceae (Fabricius) [Curculionidae, CO]

Feeding on head

Larinus rusticanus Gyllenhal [Curculionidae. CO]

Resting on head -

Larinus rusticanus Gyllenhal [Curculionidae, CO]

Reared from head

Myelois cribrella (Hübner) [Pyralidae, LE]

Orellia near colon (Meigen) [Tephritidae, DI]

do

do

do

Porphyrinia parva (Hübner) [Noctuidae, LE] -
Pyroderces argyrogrammos (Zeller) [Cosmopterigidae, LE]

do

Carthamus tinctorius L.:

Chaetorellia sp. [Tephritidae, DI]

Hylemya radicum (L.) [Anthomyiidae, DI]

do

Swept from plant

Israel.

Centaurea amara L.:

Acanthiophilus helianthi (Rossi) [Tephritidae, DI]

Reared from head

Apion detritum Rey [Curculionidae, CO]

Reared from plant

Chaetorellia sp. [Tephritidae, DI]

Reared from head

Isocolus scabiosae (Giraud) [Cynipidae, HY]

Reared from head -...-.--

Larinus planus (Fabricius) [Curculionidae, CO]

do

Lasioderma prob. obscurum Solsky [Anobiidae, CO]

do

do

Ormyrus punctiger Westwood [Ormyridae, HY]

Porphyrinia parva (Hübner) [Noctuidae, LE]

Porphyrinia parva (Hübner) [Noctuidae, LE]

d

Reared from plant

Reared from foliage -......-

Prodotis stolida (Fabricius) [Noctuidae, LE]

Reared from head -..-----

Scymnus sp. [Coccinellidae, CO] -

-.- On plant -

Sibinia attalica (Gyllenhal) [Curculionidae, CO]

Reared from head -......-

Urophora prob. quadrifasciata (Meigen) [Tephritidae, DI]

do

Do.

Do.

Do.

Do.

Do.

Do.

Do.

Do.

Do.

Do.

Do.

Do.

Do. Italy.

Do.

Do.

Do.

Do.

Do.

Do.

Do.

Do.

Do.

Do.

Do.

Do.

Do.

Do.

Centaurea caerulescens Willdenow:

Acanthiophilus helianthi (Rossi) [Tephritidae, DI]

Divales bipustulata Fabricius [Melyridae, CO]

do

Iran.

Divales sp. [Melyridae, CO]

Resting on plant

Italy.

Eudecatoma biguttata Swederus or E. submutica (Thomson)

[Eurytomidae, HY]

Eudecatoma submutica (Thomson) [Eurytomidae, HY]

Reared from head

Do.

Eurytoma robusta Mayr [Eurytomidae, HY] --

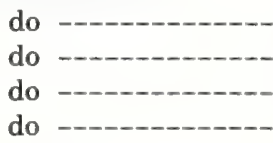

Do.

Do.

Do.

Do.

Eurytoma tibialis Boheman [Eurytomidae, HY]

Do.

See footnotes at end of table. 


\section{Table 2.-Selected weedy plants and associated insects, extent of their association, and country of origin-Continued}

\section{Plant and insect ${ }^{1}$}

\section{Insect-plant} relationship ${ }^{2}$

Country

Centaurea caerulescens Willdenow:-Continued

Ormyrus gratiosus (Foerster) [Ormyridae, HY]

Ormyrus punctiger Westwood [Ormyridae, HY]

do

do

Do.

Sibinia attalica (Gyllenhal) [Curculionidae, CO]

Reared from plant

Do.

Centaurea calcitrapa L.:

Acanthiophilus helianthi (Rossi) [Tephritidae, DI]

Reared from head

Cochylis posterana (Zeller) [Cochylidae, LE]

Reared from plant

Danacaea sp. [Melyridae, CO]

Reared from head

Eurytoma tibialis Boheman [Eurytomidae, HY] --.-----
Heliothis peltiger (Denis \& Schiffermuller) [Noctuidae, LE]

Reared from foliage

Hephathus nanus (Herrich-Schäffer) [Cicadellidae, $\mathrm{H}-\mathrm{HO}$ ]

Feeding on plant

Hysteropterum grylloides (Fabricius) [Issidae, H-HO]

Resting on foliage

Larinus longirostris Gyllenhal [Curculionidae, CO]

Reared from head

Ormyrus gratiosus (Foerster) [Ormyridae, HY] do

Reared from leaf

Phytomyza prob. horticola Goureau [Agromyzidae, DI]

Porphyrinia parva (Hübner) [Noctuidae, LE]

Reared from head

Porphyrinia parva (Hübner) [Noctuidae, LE]

Reared from plant

Pyroderces argyrogrammos (Zeller) [Cosmopterigidae, LE]

Reared from head

Urophora prob. quadrifasciata (Meigen) [Tephritidae, DI]

Swept from plant

Centaurea cineraria L.:

Drepressaria sp. [Oecophoridae, LE]

Isocolus scabiosae (Giraud) [Cynipidae, HY]

Reared from plant

Parmena sp. [Cerambycidae, CO]

Reared from head

Reared from stem

Ptinus villiger (Reitter) [Ptinidae, CO]

Reared from plant

Reared from head

Lasioderma serricorne (Fabricius) [Anobỉdae, CO]

Reared from

do $---0-1-0-0-$

Urophora prob. quadrifasciata (Meigen) [Tephritidae, DI]

Centaurea jacea L.:

Acanthiophilus helianthi (Rossi) [Tephritidae, DI]

Acanthiophilus helianthi (Rossi) [Tephritidae, DI]

Acanthiophilus helianthi (Rossi) [Tephritidae, DI]

Anthomyza gracilis Fallén [Anthomyzidae, DI] -

Bangasternus sp. [Curculionidae, CO]

do

do

do

----------

eared fror

Chaetorellia sp. [Tephritidae, DI]

Reared from head -.-------

Chaetostomella onotrophes (Loew) [Tephritidae, DI]

- Reared -..........-.

Eudecatoma biguttata Swederus or E. submutica (Thomson)

[Eurytomidae, HY] _-_-_._._.

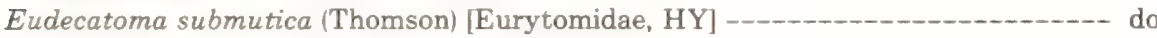

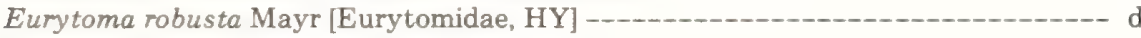

Eurytoma tibialis Boheman [Eurytomidae, HY]

Larinus planus (Fabricius) [Curculionidae, CO]

Larinus sturnus (Schaller) [Curculionidae, CO]

Lasioderma serricorne (Fabricius) [Anobiidae, CO]

Orellia falcata (Scopoli) [Tephritidae, DI]

Ormyrus punctiger Westwood [Ormyridae, HY]

Oscinella frit (L.)[Chloropidae, DI]

Polyodaspis ruficornis (Macquart) [Chloropidae, DI]

Polyodaspis ruficornis (Macquart) [Chloropidae, DI]

Reared from head

Rhinocyllus conicus (Froelich) [Curculionidae, CO]

Sarcophaga sp. [Sarcophagidae, DI]

Sitona sp. [Curculionidae, CO]

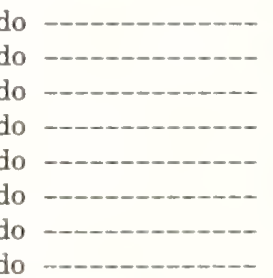

Reared from head -.-...--.

Reared from plant

Reared from head ---.-----

Reared from plant -.--.--

Reared from plant

Reared from head

Urophora quadrifasciata (Meigen) [Tephritidae, DI]

do

do

Do.

Do.

Do.

Do.

Do.

Turkey.

Italy.

Turkey.

Italy.

Do.

Do.

Yugoslavia.

Do.

Italy.

Egypt.

Italy.

Do.

Do.

Do.

Germany.

Do.

Austria.

France.

Germany.

Do.

Italy.

Do.

Germany.

Italy.

Do.

Do.

Do.

Do.

Do.

Do.

Do.

Do.

Germany.

Do.

Do.

Italy.

Do.

Germany.

Do.

Do.

See footnotes at end of table. 
Table 2.-Selected weedy plants and associated insects, extent of their association, and country of origin-Continued

\section{Plant and insect ${ }^{1}$}

\section{Insect-plant} relationship ${ }^{2}$

Country

\section{Centaurea maculosa Lamarck:}

Acanthiophilus helianthi (Rossi) [Tephritidae, DI] do

Do.

Apion onopordi Kirby [Curculionidae, CO] -

Bracon sp. [Braconidae, HY]

Corticaria sp. [Lathridiidae, CO]

Eurytoma tibialis Boheman [Eurytomidae, HY]

Meligethes incanus Sturm [Nitidulidae, CO]

Ormyrus punctiger Westwood [Ormyridae, HY]

Otiorhynchus ovatus (L.) [Curculionidae, CO]

Terellia virens (Loew) [Tephritidae, DI]

Urophora affinis (Frauenfeld) [Tephritidae, DI]

Urophora affinis (Frauenfeld) [Tephritidae, DI]

Reared from plant

Reared from head

Reared from plant

Urophora quadrifasciata (Meigen) [Tephritidae, DI]

Reared from plant

Urophora prob. quadrifasciata (Meigen) [Tephritidae, DI]

Reared from head

Urophora prob. quadrifasciata (Meigen) [Tephritidae, DI]

do

do

do

Reared from root -..--

Acalles dromedarius Boheman [Curculionidae, CO]

Acalles sp. [Curculionidae, CO] --

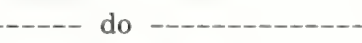

Acanthiophilus helianthi (Rossi) [Tephritidae, DI]
Attalus sp. [Melyridae, CO]

Reared from head

Reared from plant

Cnephasia sp. [Tortricidae, LE]

Reared from stem

Reared from head

[Eurytomidae, HY]

Eudecatoma submutica (Thomson) [Eurytomidae, HY]

Hypera postica (Gyllenhal) [Curculionidae, CO]

Isocolus scabiosae (Giraud) [Cynipidae, HY] - do -

Reared from plant

Reared from head

Larinus longirostris Gyllenhal [Curculionidae, CO]

do

Lasioderma serricorne (Fabricius) [Anobiidae, CO]

Rhyzobius litura (Fabricius) [Coccinellidae, CO]

Feeding on leaf

Austria.

France.

Germany.

France.

Italy.

France.

Germany.

France.

Austria.

France.

Germany.

France.

Germany.

Italy,

Do.

Do.

Do.

Do.

Do.

Do.

Do.

Do.

Do.

Do.

Do.

Centaurea napifolia L.?:

Porphyrinia parva (Hübner) [Noctuidae, LE]

Reared from plant

Do.

Centaurea nicaeensis Allioni:

Acalles sp. [Curculionidae, CO] --

Acanthiophilus helianthi (Rossi) [Tephritidae, DI]

Reared from head

Apion sp. [Curculionidae, CO]

Coleophora sp. [Coleophoridae, LE]

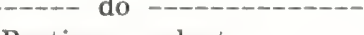

Resting on plant -.----

Reared from head

Eudecatoma biguttata Swederus or E. submutica (Thomson)

[Eurytomidae, HY]

Eudecatoma submutica (Thomson) [Eurytomidae, HY]

Eurytoma robusta Mayr [Eurytomidae, HY]

Eurytoma tibialis Boheman [Eurytomidae, HY]

Gymnetron sp. [Curculionidae, CO]

Larinus flavescens Germar [Curculionidae, CO]

Larinus longirostris Gyllenhal [Curculionidae, CO]

Larinus longirostris Gyllenhal [Curculionidae, CO]

Lasioderma serricorne (Fabricius) [Anobiidae, CO]

Leucopis sp. [Chamaemyiidae, DI]

Lonchoptera furcata (Fallén) [Lonchopteridae, DI]

do

do

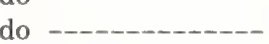

do

Reared from plant

-.- Reared -...--_..--_-

Resting on plant -.------

Reared from head --------

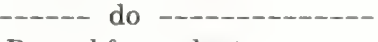

Reared from plant -..--

Mordellistena sp. [Mordellidae, CO]

Orellia near punctata (Schrank) [Tephritidae, DI]

Reared from head -..--..-

Ormyrus punctiger Westwood [Ormyridae, HY]

do ------

Urophora prob. quadrifasciata (Meigen) [Tephritidae, DI]

do
Do.

Do.

Do.

Do.

Do.

Do.

Do.

Do.

Do.

Do.

Do.

Do.

Do.

Do.

Do.

Do.

Do.

Do.

Do.

See footnotes at end of table. 
Table 2.-Selected weedy plants and associated insects, extent of their association, and country of origin-Continued

Plant and insect ${ }^{1}$

Insect-plant

relationship ${ }^{2}$

Country

Centaurea nigra L:

Chaetostomella onotrophes (Loew) [Tephritidae, DI]

do

Urophora jaceana (Hering) [Tephritidae, DI]

do

Germany.

Centaurea paniculata $\mathbf{L}$.:

Acanthiophilus helianthi (Rossi) [Tephritidae, DI]

do

France.

Centaurea phrygia L.:

Chaetostomella onotrophes (Loew) [Tephritidae, DI]

do

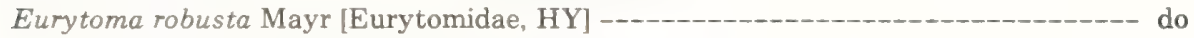

Eurytoma tibialis Boheman [Eurytomidae, HY] ---

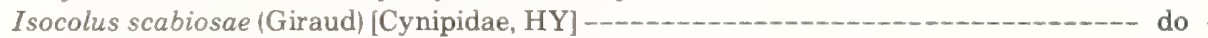

Larinus australis Capiomont [Curculionidae, CO]

Centaurea repens $\mathbf{L}$.:

Acanthiophilus helianthi (Rossi) [Tephritidae, DI]

Swept from plant

Attalus sp. [Melyridae, CO]

Bruchidius quinqueguttatus (Olivier) complex [Bruchidae, CO]

do --n--------

Circulifer opacipennis (Lethierry) [Cicadellidae, H-HO]

do -------------

Clytra atraphaxidis Pallas or near [Chrysomelidae, CO]

do - - - -

Probably Coptocephala sp. [Chrysomelidae, CO]

Deltocephalus sp. [Cicadellidae, $\mathrm{H}-\mathrm{HO}]$

do

Eumerus strigatus Falién [Syrphidae, DI]

Handianus sp. [Cicadellidae, $\mathrm{H}-\mathrm{HO}$ ]

Holcostethus sp. [Pentatomidae, H-HE]

Hyalesthes obsoletus Signoret [Cixiidae, H-HO]

Oliarus barajus Dlabola [Cixiidae, $\mathrm{H}-\mathrm{HO}$ ]

Spermophagus sericeus (Geoffroy) [Bruchidae, CO]

Tettigometra griseola Fieber [Tettigometridae, H-HO]

Tettigometra sp. [Tettigometridae, $\mathrm{H}-\mathrm{HO}$ ]

Thaumatomyia sulcifrons (Becker) [Chloropidae, DI]

Timia xanthaspis (Loew) [Otitidae, DI]

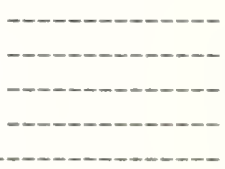

Do.

Germany.

Do.

Italy.

Germany.

Austria.

Iran.

Do.

Do.

Do.

Do.

Do.

Do.

Do.

Do.

Do.

Do.

Do.

Do.

Do.

Do.

Do.

Do.

Centaurea scabiosa L:

Chaetostomella onotrophes (Loew) [Tephritidae, DI]

Reared from head

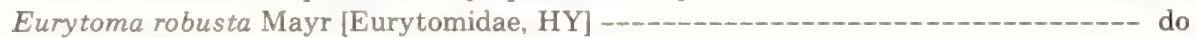

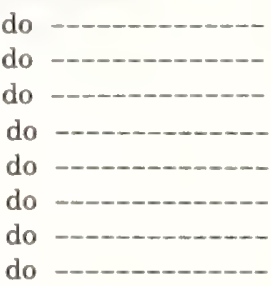

Eurytoma tibialis Boheman [Eury tomidae, HY] -

Isocolus scabiosae (Giraud) [Cynipidae, HY] -

Larinus near leuzeae Fabre [Curculionidae, CO]

Orellia colon (Meigen) [Tephritidae, DI]

Orellia sp. [Tephritidae, DI]

Urophora cuspidata (Meigen) [Tephritidae, DI]

Urophora prob. quadrifasciata (Meigen) [Tephritidae, DI]

do

Resting on flower

Reared from head

Germany.

Do.

Do.

Do.

Do.

Do.

Do.

Do.

Do.

Centaurea sphaerocephala L.:

Orellia punctata (Schrank) [Tephritidae, DI]

Psilothrix sp. [Melyridae, CO]

Pyroderces argyrogrammos (Zeller) [Cosmopterigidae, LE]

Centaurea sp.:

Acalles sp. [Curculionidae, CO] -

Reared from plant

Caenacis sp. [Pteromalidae, HY]

Reared from head

taly.

Do.

Do.

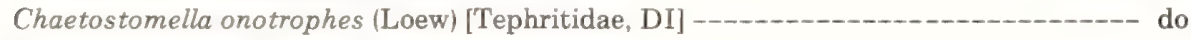

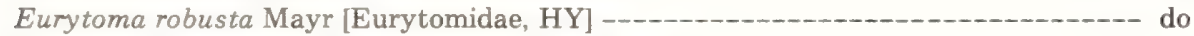

do --_-_-_-_-_---

Larinus prob. australis Capiomont [Curculionidae, CO]

do

Rhinocyllus conicus (Froelich) [Curculionidae, CO]

Terellia serratulae (L.) [Tephritidae, DI]

Do.

Do.

Do.

Do.

Do.

Do.

Do.

See footnotes at end of table. 
Table 2.-Selected weedy plants and associated insects, extent of their association, and country of origin-Continued

Plant and insect ${ }^{1}$

Insect-plant

relationship ${ }^{2}$

Country

Cirsium arvense (L.) Scopoli:

Altica carduorum (Guérin-Ménevillé) [Chrysomelidae, CO]

Apion detritum Rey [Curculionidae, CO] -

Bruchidius sp. [Bruchidae, CO]

Reared from stem

Reared from head

Cassida prob. vibex L. [Chrysomelidae, CO]

Reared from foliage

Ceutorhynchus litura (Fabricius) [Curculionidae, CO]

Resting on plant

Chaetostomella onotrophes (Loew) [Tephritidae, DI]

Reared from plant

Cleonus piger (Scopoli) [Curculionidae, CO]-

Reared from root

Cochylis near hybridella (Hübner) [Cochylidae, LE]

Reared from head

Corticaria sp. [Lathridiidae, CO]

Epuraea sp. [Nitidulidae, CO]

Reared from plant

Eurytoma robusta Mayr [Eurytomidae, HY]

Reared from head

Eurytoma tibialis Boheman [Eurytomidae, HY]

do

Eurytoma tibialis Boheman [Eurytomidae, HY]

Larinus planus (Fabricius) [Curculionidae, CO]

Feeding on plant

Larinus planus (Fabricius) [Curculionidae, CO]

Reared from head

Larinus planus (Fabricius) [Curculionidae, CO]

Resting on plant
Reared from head

Larinus turbinatus Gyllenhal [Curculionidae, CO]

Larinus turbinatus Gyllenhal [Curculionidae, CO]

do

Larinus turbinatus Gyllenhal [Curculionidae, CO]

do

Larinus turbinatus Gyllenhal [Curculionidae, CO]

Resting on plant

Larinus turbinatus Gyllenhal [Curculionidae, CO]

Lema cyanella L. [Chrysomelidae, CO]

Meligethes sp. [Nitidulidae, CO]

Ormyrus punctiger Westwood [Ormyridae, HY]

Reared from plant

Otiorhynchus sp. [Curculionidae, CO]

Reared from head

Philaenus spumarius (L.) [Cercopidae, H-HO]

Reared from plant

Phora sp. [Phoridae, DI]

Pyroderces argyrogrammos (Zeller) [Cosmopterigidae, LE]

Sitona sp.[Curculionidae, CO]

Sphaeroderma rubidum (Graëlls) [Chrysomelidae, CO]

Reared from plant

Reared from head

Sphaeroderma testaceum L. [Chrysomelidae, CO]

Stictopleurus punctatonervosus (Goeze) [Rhopalidae, $\mathrm{H}-\mathrm{HE}$ ]

Tychius quinquepunctatus (L.) [Curculionidae, CO]

Urophora stylata (Fabricius) [Tephritidae, DI]

Resting on head

Swept from plant

Reared from head

France.

Italy.

Germany.

Yugoslavia.

Switzerland.

Germany.

Italy.

Yugoslavia.

Germany.

Do.

Do.

Do.

Yugoslavia.

Germany.

Do.

Switzerland.

Spain.

Italy.

Germany.

Yugoslavia.

Germany.

France.

England.

Italy.

Yugoslavia.

Germany.

Italy.

Germany.

Italy.

Germany.

Switzerland.

Do.

Germany.

Italy.

Yugoslavia..

Cirsium odontolepis Boissier:

Porphyrinia purpurina (Denis \& Schiffermüller) [Noctuidae, LE]

Reared from plant

Italy.

Cirsium vulgare (Savi) Tenore:

Adscita sp. [Zygaenidae, LE]

Altica carduorum (Guérin-Ménevillé [Chrysomelidae, CO]

do

- Feeding on leaf --

Do.

Do.

Do.

Apion sp. [Curculionidae, $\mathrm{CO}$ ]

Reared from crown

France.

Cleonus morbillosus (Fabricius) [Curculionidae, CO]

Reared from root

Germany.

Cleonus piger (Scopoli) [Curculionidae, CO]

Reared from root - - - - - -

Italy.

Epiblema near pflugiana (Fabricius) [Tortricidae, LE]

Reared from stem

Do.

Eupithecia sp. [Geometridae, LE]

Reared from head

Eurytoma robusta Mayr [Eurytomidae, HY]

do

do

Eurytoma tibialis Boheman [Eurytomidae, HY]

Homeosoma near nebulella (Denis \& Schiffermüller) [Pyralidae, LE]

do

do

do

Larinus sturnus (Schaller) [Curculionidae, CO]

do

France.

Italy.

France.

Italy.

Germany.

France.

Italy.

See footnotes at end of table. 
Table 2.-Selected weedy plants and associated insects, extent of their association, and country of origin-Continued

Plant and insect ${ }^{1}$

\section{Insect-plant}

relationship ${ }^{2}$

Country

Cirsium vulgare (Savi) Tenore:-Continued

Larinus turbinatus Gyllenhal [Curculionidae, CO]

Lixus algirus (L.) [Curculionidae, CO]

do -

Do.

Lixus elongatus (Goeze) [Curculionidae, CO]

Reared from stem -

do

Phyllobius sp. [Curculionidae, CO]

Resting on leaf

Resting on plant

Reared from plant

Sitona sp. [Curculionidae, CO]

Sitona sp. [Curculionidae, CO]

from head

Terellia longicauda (Meigen) [Tephritidae, DI]

Reared from head

Urophora stylata (Fabricius) [Tephritidae, DI]

do

Urophora stylata (Fabricius) [Tephritidae, DI]

Cirsium sp.:

Agapanthia dahli (Richter) [Cerambycidae, CO]

Lasioderma baudii (Schilsky) [Anobiidae, CO]

Urophora stylata (Fabricius) [Tephritidae, DI]

Reared from head

Resting on head

Do.

Do.

Do.

Do.

Germany.

Italy.

France.

Germany.

Italy.

Cynara cardunculus $\mathrm{L}$.:

Voria ruralis (Fallén) [Tachinidae, DI]

Reared from noctuid larva

Cynara scolymus $\mathbf{L}$.:

Acalles diocletianus (Germar) [Curculionidae, CO]

Acalles dromedarius Boheman [Curculionidae, CO]

Acanthiophilus helianthi (Rossi) [Tephritidae, DI]

Biosteres carbonaris (Nees) [Braconidae, HY]

Bracon sp. [Braconidae, HY]

Cacoecimorpha pronubana (Hübner) [Tortricidae, LE]

Drosophila funebris (Fabricius) [Drosophilidae, DI]

Gastroidea polygoni (L.) [Chrysomelidae, CO]

Homoeosoma sp. [Pyralidae, LE]

Larinus scolymi (Olivier) [Curculionidae, CO]

Larinus scolymi (Olivier) [Curculionidae, CO]

Lasioderma baudii (Schilsky) [Anobiidae, CO]

Melitaea cinxia (L.) [Nymphalidae, LE]

Prodotis stolida (Fabricius) [Noctuidae, LE]

Pyroderces argyrogrammos (Zeller) [Cosmopterigidae, LE]

Sitona sp. [Curculionidae, CO]

Sitona sp. [Curculionidae, CO]

Sitophilus sp. [Curculionidae, CO] -

Sphaeroderma rubidum (Graëlls) [Chrysomelidae, CO]

Reared from head

Reared from plant

Reared from head

Parasitic on anthomyiid -...-.

Reared from unknown insect ---

Reared from head

do

Resting on leaf

Reared from head

Resting on plant

Reared from plant

On plant

Reared from foliage

Reared from plant

Reared from head

- Resting on head -..-.-.-.-.

Reared from head -...-..-

Feeding on foliage --------

Subcoccinella vigintiquattuorpunctata (L.) [Coccinellidae, CO]

- On plant

Reared from head

Austria.

Italy.

Do.

Do.

Do.

Do.

Do.

Do.

Do.

Do.

Do.

Do.

Do.

Do.

Do.

Do.

Do.

Do.

Do.

Do.

Do.

Do.

Do.

Do.

Terellia fuscicornis (Loew) [Tephritidae, DI]

Reared from stem

Do.

Cytisus scoparius (L.) Link:

Agrilus biguttatus (Fabricius) [Buprestidae, CO] -

Swept from plant

Do.

Agrilus biguttatus (Fabricius) [Buprestidae, CO]

- From branch

France.

Anaspis sp. [Mordellidae, CO]

Reared from seed -.--

Bruchidius seminarius (L.) [Bruchidae, CO]

Chrysomela or near [Cheysomelidae, CO]

Leucoptera spartifoliella (Hübner) [Lyonetiidae, LE]

Leucoptera spartifoliella (Hübner) [Lyonetiidae, LE]

Phloeotribus rhododactylus (Marsham) [Scolytidae, CO]

$?$

Italy.

France.

Do.

Italy.

Phytodecta olivacea Forster [Chrysomelidae, CO]

Reared from dead branch -..--

France.

Do.

Cytisus sp.:

Apion near compactum Desbrochers [Curculionidae, CO]

See footnotes at end of table. 
Table 2.-Selected weedy plants and associated insects, extent of their association, and country of origin-Continued

\section{Plant and insect ${ }^{1}$}

\section{Insect-plant} relationship ${ }^{2}$

Echinops sphaerocephalus L.:

Eurytoma robustus Mayr or E. tibialis Boheman [Eurytomidae, HY]

Sitona sp. [Curculionidae, CO]

Reared from head

Italy.

Strophomorphus porcellus (Schönherr) [Curculionidae, CO]

Euphorbia esula L.:

Chamaesphecia empiformis (Esper) [Sesiidae, LE]

Reared from crown

Do.

Euphorbia sp.:

Hyles euphorbiae (L.) [Sphingidae, LE]

Reared from roots

Austria.

Galactites tomentosa Moench:

Agapanthia cardui (L.) [Cerambycidae, CO]

Reared from foliage

Italy.

Agapanthia dahli (Richter) [Cerambycidae, CO] -

Resting on plant

Ceutorhynchidius horridus (Panzer) [Curculionidae, CO]

- do

do -

Ceutorhynchidius horridus (Panzer) [Curculionidae, CO] ----

Reared from crown ---.---

Ceutorhynchus trimaculatus (Fabricius) [Curculionidae, CO]

- Feeding on leaf -

Ceutorhynchus trimaculatus [Curculionidae, $\mathrm{CO}]$

Reared from crown

Pyrodercés argyrogrammos (Zeller) [Cosmopterigidae, LE]

Reared from head

France.

Italy.

Do.

Do.

Do.

Do.

Do.

Genista sagittalis L.:

Apion near formaneki Wagner [Curculionidae, CO]

Germany.

Genista sp.:

Apion sp. [Curculionidae, CO]

Do.

Hypericum sp.:

Chrysolina hyperici Forster [Chrysomelidae, CO]

Feeding on foliage

Italy.

Linaria dalmatica (L.) Miller:

Brachypterolus pulicarius (L.) [Nitidulidae, CO]

Resting on plant

Calophasia casta (Borkhausen) [Noctuidae, LE]

Reared from leaves

Chrysolina gypsophilae Küster [Chrysomelidae, CO]

Resting on plant

Eurydema ornatum L. [Pentatomidae, H-HE]

Reared from seed

Nezara virdula [Pentatomidae, $\mathrm{H}-\mathrm{HE}]$

-

Podabrus sp. [Cantharidae, CO] -

Stragmatophora serratella (Treitschke) [Cosmopterigidae, LE]

Resting on plant

Resting on foliage

Resting on flower

Feeding on stem

Resting on plant

Linaria fastigiata Chavannes:

Brachypterolus pulicarius (Linnaeus) [Nitidulidae, CO]

Gymnetron sp. [Curculionidae, CO]

Phytoecia sp. [Cerambycidae, CO]

Resting do

Brachypterolus pulicarius (L.) [Nitidulidae, CO]

do

do

Brachypterolus sp. [Nitidulidae, $\mathrm{CO}]$

Bruchus dentipes Baudi [Bruchidae, $\mathrm{CO}]$

Gymnetron sp. [Curculionidae, $\mathrm{CO}$ ]

Gymnetron sp. [Curculionidae, CO]

do

do

Reared from plant

Oscinella sp. [Chloropidae, DI]

do - -

Phytoecia sp. [Cerambycidae, CO]

Feeding on stem

Yugoslavia.

Do.

Do.

Italy.

Do.

Do.

Do.

Do.

Iran.

Do.

Do.

Linaria grandiflora Desfontaines:

Brachypterolus pulicarius (L.) [Nitidulidae, CO]

Resting on plant

Bruchus brachialis Fabricius [Bruchidae, $\mathrm{CO}$ ]

Resting on plant

Platypalpus sp. [Empididae, DI]

Reared from plant

Do.

Do.

Do.

See footnotes at end of table. 
Table 2.-Selected weedy plants and associated insects, extent of their association, and country of origin-Continued

Plant and insect ${ }^{3}$

Insect-plant

relationship ${ }^{2}$

Country

Linaria heterophylla Desfontaines:

Brachypterolus pulicarius (L.) [Nitidulidae, CO] -

Gymnetron netum (Germar) [Curculionidae, CO]

Resting on plant

Reared from seed pod

Italy.

Julistus sp. [Melyridae, CO]

Resting on plant

Do.

Linaria kurdica Boissier \& Hohenacker:

Brachypterolus pulicarius (L.) [Nitidulidae, CO]

Brachypterolus pulicarius (L.) [Nitidulidae, CO]

do

Phytoecia sp. [Cerambycidae, CO]

Resting on bud -

Resting on stem

Do.

Linaria lineolata Boissier:

Brachypterolus pulicarius (L.) [Nitidulidae, CO]

Resting on plant

Gymnetron sp. [Curculionidae, $\mathrm{CO}$ ]

Linaria repens (L.) Miller:

Gymnetron sp. [Curculionidae, CO]

Stagmatophora serratella (Treitschke) [Cosmopterigidae, LE],

Reared from seed

Stagmatophora serratella (Treitschke) [Cosmopterigidae, LE]

Resting on plant

Reared from root

-

Linaria vulgaris Miller:

Attalus sp. [Melyridae, CO] -

Reared from plant

Brachypterolus pulicarius (L.) [Nitidulidae, CO]

Brachypterolus pulicarias (L.) [Nitidulidae, CO]

Brachypterolus pulicarius (L.) [Nitidulidae, CO]

Brachypterolus sp. [Nitidulidae, CO]

----- do

Resting on flowers

Resting on plant

Reared from plant

Brachypterolus sp. [Nitidulidae, CO]

Resting on foliage

Reared from plant material -..-

Bracon sp. [Braconidae, $\mathrm{HY}]$

- Reared from plant -.--.---

Chelonus sp. [Braconidae, HY]

Reared from Stagmatophora --serratella larva in root.

Chrysolina sp. [Chrysomelidae, CO] -- In soil near root --

Cymus glandicolor Hahn [Lygaeidae, H-HE]

- Resting on plant ---- -

Dacnusa leptogaster Haliday [Braconidae, HY]

Reared from plant material -----

Probably Danacaea sp. [Melyridae, CO]

Reared from plant

Linaria vulgaris Miller:

Probably Danacaea sp. [Melyridae, CO]

Resting on plant

Eupithecia linariata (Denis \& Schiffermuiller) [Geometridae, LE]

Reared from flower

Gymnetron antirrhini (Paykull) [Curculionidae, CO]

Reared from seed

Gymnetron antirrhini (Paykull) [Curculionidae, CO]

Resting on flower

Reared from pod

Reared from root

Gymnetron linariae (Panzer) [Curculionidae, CO]

Reared from seed pod

Gymnetron netum (Germar) [Curcul

Reared from root gall

Gymnoscelis pumilata (Hübner) [Geometridae, LE]

Reared from plant

Resting on plant

Holcostethus strictus (Fabricius) [Pentatomidae,
Mecinus janthinus (Germar) [Curculionidae, CO]

- do

Melitaea didyma (Esper) [Nymphalidae, LE]

Reared from foliage -......-

Palpita unionalis (Hübner) [Pyralidae, LE]

Reared from plant

Stagmatophora serratella (Treitschke) [Cosmopterigidae, LE]

Reared from root

Turkey.

Do.

Do.

Iran.

Do.

France.

Do.

Do.

Italy.

France.

Italy.

Yugoslavia.

Italy.

France.

Do.

Italy.

Do.

Do.

Do.

France.

Italy.

Do.

Do.

Do.

Do.

France.

Do.

Italy,

Do.

Do.

Do.

Do.

Do.

Do.

Do.

Linaria sp.:

Adelphocoris lineolatus (Goeze) [Miridae, H-HE]

On leaf

Adelphocoris sp. [Miridae, H-HE]

do -

Turkey.

Do.

Adonia variegata (Goeze) [Coccinellidae, $\mathrm{CO}$ ]

do - on plant.

Do.

Alloeonotus fulvipes (Scopoli) [
Anaphes sp. [Mymaridae, HY]

Resting on plant --..--

Anthicus sp. [Anthicidae, CO]

Ex flower

On flower

Yugoslavia.

Turkey.

Do.

See footnotes at end of table. 
Table 2.-Selected weedy plants and associated insects, extent of their association, and country of origin-Continued

\section{Insect-plant}

relationship ${ }^{2}$

Country

Linaria sp.:-Continued

Anthocoris nemorum (L.) [Anthocoridae, H-HE]

On leaf

Do.

Apion sp. [Curculionidae, CO] -

Aplocnemus sp. [Melyridae, CO]

Resting on plant

Brachycoleus decolor Reuter [Miridae, H-HE]

On leaf

Do.

Brachypterolus pulicarius (L.) [Nitidulidae, CO]

Brachypterolus pulicarius (L.) [Nitidulidae, CO]

Brachypterolus pulicarius (L.) [Nitidulidae, CO]

Bracon near osculator Nees [Braconidae, HY]

- do

Feeding on flowers

Reared from plant material ----

Calophasia casta (Borkhausen) [Noctuidae, LE]

On flower

Pupa on plant

Camponotus lateralis (Olivier) [Formicidae, HY]

On leaf

Cardiophorus near niggerinus Erichson [Elateridae, CO]

do

do

Carpocoris lunulatus (Goeze) [Pentatomidae, H-HE]

Carpocoris mediterraneus Taminini [Pentatomidae, H-HE]

Carpocoris pudicus (Poda) [Pentatomidae, H-HE] -

Cataglyphis near cursor (Fonscolombe) [Formicidae, HY]

Chrysolina variolosa Pethena [Chrysomelidae, CO]

Chrysolina sp. [Chrysomelidae, CO]

Chrysolina sp. [Chrysomelidae, CO]

Chrysopa near carnea Stephens [Chrysopidae, NE]

- do

do

do

Reared from foliage

Feeding on leaf

Cicadatra near hyalina (Fabricius) [Cicadidae, H-HO]

On leaf

On stem

Clytra laeviuscula Ratzeburg or near [Chrysomelidae, CO]

On leaf

Coccinella quinquepunctata L. [Coccinellidae, CO]

Coccinella septempunctata L. [Coccinellidae, CO]

Colotes sp. [Melyridae, CO]

Corizus hyoscyami Linnaeus [Rhopalidae, H-HE]

Probably Danacaea sp. [Melyridae, CO]

On stem

Probably Danacaea sp. [Melyridae, CO]

On flower

On leaf

On stem

Dictyla echii (Shrank) [Tingidae, H-HE]

On leaf

Dolycoris baccarum (L.) [Pentatomidae, H-HE]

- do

do

Entedon sp. [Eulophidae, HY]

Eupelmus sp. [Eupelmidae, HY]

On flower

Eurygaster maura (L.) [Pentatomidae, H-HE]

On leaf

Eurytoma sp. [Eurytomidae, HY]

Ex seed pod

On seed pod

Eurytoma sp. [Eurytomidae, HY]
Eysarcoris inconspicuus (Herrich-Schäffer) [Pentatomidae, H-HE]

Resting on plant

Formica near nasuta Nylander [Formicidae, HY]

Formica sp. [Formicidae, HY]

On stem

Heliothis sp. [Noctuidae, LE]

Leucopis sp. [Chamaemyiidae, DI]

- do

On plant

do

Leucocelis sp. [Scarabaeidae, CO]

On flower

Lygaeus equestris (L.) [Lygaeidae, H-HE]

On leaf

Lygus pratensis (L.) [Miridae, $\mathrm{H}-\mathrm{HE}$ ]

do

do

lo

Mecinus sp. [Curculionidae, $\mathrm{CO}$ ]

Melitaea didyma (Esper) [Nymphalidae, LE]

Reared from plant

Microplitis poss, scrophulariae Szelenyi [Braconidae, HY]

On flower

Microplitis poss. variipes Ruthe [Braconidae, HY]

Mylabris apicipennis Reiche [Meloidae, CO]

Mylabris sp. [Meloidae, CO]

Nemotelus sp. \#1 [Stratiomyidae, DI]

Nemotelus sp. \#2 [Stratiomyidae, DI]

- do

do

On leaf

Reared from stem

Nemotelus sp. \#2 [Stratiomyidae, DI]

On flower
Do.

Bulgaria.

Italy.

Turkey.

Italy.

Turkey.

Do.

Do.

Do.

Do.

Do.

Italy.

Turkey.

Bulgaria.

Turkey.

Do.

Do.

Yugoslavia.

Turkey.

Yugoslavia..

Turkey.

Do.

Yugoslavia.

Turkey.

Do.

Do.

Do.

Yugoslavia.

Turkey.

Do.

Do.

Italy.

Turkey.

Do.

Do.

Do.

Yugoslavia.

Turkey.

Do.

Do.

Do.

Do.

Do.

Do.

Do.

Do.

Do

Do.

See footnotes at end of table. 
Table 2.-Selected weedy plants and associated insects, extent of their association, and country of origin-Continued

Plant and insect ${ }^{1}$

Insect-plant relationship ${ }^{2}$

Country

Linaria sp.:-Continued

Omophulus sp. [Alleculidae, CO]

Paragus sp. [Syrphidae, DI]

Resting on plant

Phyllotreta sp. [Chrysomelidae, CO]

- On flower

Italy.

Phytomyza prob. horticola Goureau [Agromyzidae, DI]

- Resting on foliage

- Resting on leaf mine

Plutella xylostella (L.) [Plutellidae, LE] -

Reared from pupa on leaf --.--

Podonta aubei Mulsant [Alleculidae, CO]

On leaf

Polymerus sp. [Miridae, $\mathrm{H} \cdot \mathrm{HE}$ ]
Rhinocoris iracundus Poda [Reduviidae, $\mathrm{H}-\mathrm{HE}$ ]

- do

Rhopalus sp. [Rhopalidae, H-HE]

do

Scymnus sp. [Coccinellidae, CO]

On flower

Scymnus (s.str.) sp. [Coccinellidae, CO]

On leaf

Spilostethus saxatilis (Scopoli) [Lygaeidae, H-HE]

Spintherus sp. [Pteromalidae, HY]

- do

Spintherus sp. [Pteromalidae, HY]

On seed pod

Stagmatophora serratella (Treitschke) [Cosmopterigidae, LE]

- On flower

Subcoccinella vigintiquattuorpunctata (L.) [Coccinellidae, CO]

Tapinoma sp. [Formicidae, HY]

Reared from root

Telenomus sp. [Scelionidae, HY]

On leaf

On stem

Ex flower

Tettigonia caudata (Charpentier) [Tettigonidae, OR]

On leaf

Onopordum acanthium L.:

Lixus cardui Olivier [Curculionidae, CO]

Reared from stem

Turkey.

Do.

Do.

Do.

Do.

Do.

Do.

Do.

Yugoslavia.

Turkey.

Do.

Do.

Do.

Do.

Do.

Do.

Do.

Do.

Onopordum tauricum Willdenow:

Apion onopordi Kirby [Curculionidae, CO]

Reared from crown

Cercopis vulnerata Rossi [Cercopidae, H-HO]

Resting on plant

Reared from root

Crepidodera ferruginea (Scopoli) or near [Chrysomelidae, CO]

Resting on plant

Deraeocoris schach (Fabricius) [Miridae, H-HE]

Larinus australis Capiomont [Curculionidae, CO]

Larinus cynarae (Fabricius) [Curculionidae, $\mathrm{CO}$ ] do

do

Resting on stem

Reared from stem

Reared from head

Resting on plant

Reared from head

Orellia lappae (Cederhjelm) [Tephritidae, DI]

Philaenus spumarius (L.) [Cercopidae, H-HO]

Sitona sp. [Curculionidae, CO]

Tephritis postica (Loew) [Tephritidae, DI] do

Reared from head

Acanthiophilus helianthi (Rossi) [Tephritidae, DI]

Cochylis posterana (Zeller) [Cochylidae, LE]

Cryptocephalus hypochoeridis (L.) [Chrysomelidae, CO]

do

Dolycoris baccarum (L.) [Pentatomidae, H-HE]

Resting on leaves

Reared from head

Resting on leaves

Glaphyrus sp. [Scarabaeidae, CO]

Resting on plant

Graphosoma lineata (L.) [Podopidae, H-HE] -

Resting on stem -

Larinus cynarae (Fabricius) [Curculionidae, CO] -

Larinus latus (Herbst) [Curculionidae, CO]

Resting on plant

Reared from stem

Resting on foliage

Resting on leaves

Reared from stem

Reared from head

Resting on plant

Reared from head

do

do

Tephritis postica (Loew) [Tephritidae, DI]

Terellia near virens (Loew) [Tephritidae, DI]

See footnotes at end of table. 
Table 2.-Selected weedy plants and associated insects, extent of their association, and country of origin-Continued

Plant and insect $t^{1}$ Insect-plant relationship ${ }^{2}$ Country

Quercus pubescens Willdenow:

Bruchus brachialis Fabricius [Bruchidae, $\mathrm{CO}$ ]

Salsola kali L.:

Coleophora sp. [Coleophoridae, LE] Ex plant

Egypt.

Gymnancyla canella (Denis \& Schiffermüller) [Pryralidae, LE]

Reared from plant

Italy.

Salsola sp.:

Cryptocephalus populi Suffrian or near [Chrysomelidae, CO]

Do.

Geocoris megacephalus siculus (Fieber) [Lygaeidae, H-HE]

Do.

Salvai aethiopis $\mathbf{L}$.:

Meligethes incanus Sturm [Nitidulidae, CO]

Phrydiuchus tau Warner [Curculionidae, CO]

Turkey.

Salvia argentea L.:

Phrydiuchus spilmani Warner [Curculionidae, CO]

Resting on plant

Yugoslavia.

Salvia limbata C. A. von Meyer:

Heliothis peltiger (Denis \& Schiffermüller) [Noctuidae, LE]

Reared from foliage

Italy.

Salvia palaestina Bentham:

Cynthia cardui (L.) [Nymphalidae, LE] do

Salvia verbenaca $L$.

Phytoecia pustulata (Schrank) [Cerambycidae, CO]

Phytometra sp. [Noctuidae, LE]

Reared from foliage

Italy.

Salvia virgata Jacquin:

Sphragisticus nebulosus (Fallén) [Lygaeidae, H-HE]

Do.

Salvia sp.:

Chrysolina grossa (Fabricius) [Chrysomelidae, CO]

Do.

Scrophularia canina L.:

Ancyrosoma leucogrammum (Gmelin) [Podopidae, H-HE]

Resting under plant

Berytinus sp. [Berytidae, H-HE]

Heterogaster urticae (Fabricius) [Lygaeidae, H-HE]

Mesocerus marginatus (L.) ? [Coreidae, H-HE]

Phymata crassipes (Fabricius)? [Phymatidae, H-HE]

do - - -

Spermophagus sericeus (Geoffroy) [Bruchidae, CO] -

do

do

do

do

Turkey.

Strobilotoma typhaecornis (Fabricius) [Coreidae, H-HE]

Do.

Do.

Do.

Do.

Do.

Do.

Do.

Senecio aquaticus Hill:

Agapanthia prob. dahli (Richter) [Cerambycidae, CO]

Reared from stem

Do.

Alophus nictitans Boheman [Curculionidae, CO]

Feeding on root

Do.

Bracon sp. [Braconidae, HY]

Prob. ex Agapanthia larva ----

Chaetocnema prob. conducta Motschulsky [Chrysomelidae, CO]

- Swept from plant

Chlorissa sp. [Geometridae, LE]

Reared from plant

Cochylis prob. atricapitana (Stephens) [Cochylidae, LE]

Reared from crown -......

Eupithecia centaurearia (Denis \& Schiffermüller) [Geometridae, LE]

Reared from plant -......-.

Gastroidea polygoni (L.) [Chrysomelidae, CO]

In soil near roots ---------

Liriomyza strigata (Meigen) [Agromyzidae, DI]

Reared from leaf

Lixus punctiventris Boheman [Curculionidae, $\mathrm{CO}$ ]

Reared from crown \& roots ---

Longitarsus dorsalis (Fabricius) [Chrysomelidae, CO]

- Feeding on leaf --.------

Longitarsus ganglbaueri Heikertinger [Chrysomelidae, CO]

Feeding on foliage

Longitarsus prob. ganglbaueri Heikertinger [Chrysomelidae, CO]

Swept from plant

Longitarsus jacobaea Waterhouse [Chrysomelidae, CO]

Resting on foliage

Longitarsus prob. jacobaea Waterhouse [Chrysomelidae, CO]

Swept from plant

Longitarsus near tabidus (Fabricius) [Chrysomelidae, CO]

Reared from roots

Melanagromyza dettmeri Hering [Agromyzidae, DI]

Reared from stem

Melanagromyza near tripolii Spencer [Agromyzidae, DI]

do

Do.

Do.

Do.

Do.

Do.

Do.

Do.

Do.

Do.

Do.

Do.

Do.

Do.

Do.

Do.

Do.

See footnotes at end of table. 


\section{Table 2.-Selected weedy plants and associated insects, extent of their association, and country of origin-Continued}

Plant and insect ${ }^{1}$

Insect-plant

relationship ${ }^{2}$

Country

Senecio aquaticus Hill:-Continued

Otiorhynchus rugostriatus (Goeze) [Curculionidae, CO]

Reared from roots

Do.

Oulema melanopus (L.) [Chrysomelidae, CO] Resting on leaves -.....---

Philaenus spumarius (L.) [Cercopidae, H-HO]

Reared from stem -.....--

Philaenus spumarius (L.) [Cercopidae, H-HO]

Resting on foliage -.....--

Philaenus spumarius (L.) [Cercopidae, H-HO]

Reared from foliage --.----

Phycitodes carlinella (Heinemann) [Pyralidae, LE]

Reared from plant

Sitona puncticollis Stephens [Curculionidae, CO]

do

Do.

Do.

Do.

Do.

Do.

Do.

Senecio ercifolius L.:

Cacoecimorpha pronubana (Hübner) [Tortricidae, LE]

Reared from foliage

Do.

Senecio jacobaea L:

Apanteles sp. [Braconidae, HY]

Reared from plant

Bracon sp. [Braconidae, HY]

Reared from plant material -.--

Bufolucilia silvarum (Meigen) [Calliphoridae, DI] Reared

- Reared from crown -..---.-

Cochylis prob. atricapitana (Stephens) [Cochylidae, LE]

Reared from agromyzid -----

Diglyphosema sp. [Cynipidae, HY]

Reared from head

Eucosma campoliliana (Denis \& Schiffermüller) ? [Tortricidae, LE]

Reared from plant

Eumerus sp. [Syrphidae, DI]

Feeding in root

Eupithecia absinthiata (Clerck) [Geometridae, LE]

Eupithecia centaurearia (Denis \& Schiffermuiller) [Geometridae, LE]

Reared from plant --------

Eutrias sp. [Cynipidae, HY]

Exartema sp. [Tortricidae, LE]

red from Diptera pupa

Fannia sp. [Muscidae, DI]

Reared from plant -...--

Hylemya brassicae (Wiedemann) [Anthomyiidae, DI]

Swept from plant

Hylemya cinerella (Fallén) [Anthomyidae, DI] -

Hylemya coarctata (Fallén) [Anthomyiidae, DI]

do

do

Hylemya platura (Meigen) [Anthomyiidae, DI] -

- do

Hylemya pullula (Zetterstedt) [Anthomyiidae, DI]

Reared from plant

Swept from plant

Hylemya radicum (L.) [Anthomyiidae, DI]

Hylemya seneciella (Meade) [Anthomyidae, DI]

Lasioderma baudii (Schilsky) [Anobiidae, CO]

Reared from head

Reared from plant

Liriomyza latigenis (Hendel) [Agromyzidae, DI] - do

Lixus punctiventris Boheman [Curculionidae, CO]

-

Lixus punctiventris Boheman [Curculionidae, CO]

Reared from crown \& roots -.--

Longitarsus prob. ganglbaueri Heikertinger [Chrysomelidae, CO] -

Swept from plant .........

Longitarsus prob. jacobaea Waterhouse [Chrysomelidae, CO]

Longitarsus prob. jacobaea Waterhouse [Chrysomelidae, CO]

Longitarsus sp. [Chrysomelidae, CO]

- - - do

Reared from root -

Longitarsus sp. [Chrysomelidae, CO]

In soil under plant

Melanagromyza aeneoventris (Fallén) [Agromyzidae, DI]

Resting on plant

Reared from stem

Melanagromyza eupatorii Spencer [Agromyzidae, DI]

do

Olibrus corticalis (Panzer) [Phalacridae, CO]

Ophiomyia senecionina Hering [Agromyzidae, DI]

Reared from plant

Opius sp. [Braconidae, HY]

- do

Orgilus sp. [Braconidae, HY]

Oulema melanopus (L.) [Chrysomelidae, CO]

Reared from crown \& roots -.--

Oxyna nebulosa (Wiedemann) [Tephritidae, DI]

Reared from plant

- Resting on head -

Resting on stem

Pantomorus cervinus (Boheman) [Curculionidae, CO]

Reared from plant

Paroxyna prob. tessellata (Loew) [Tephritidae, DI]

Probably Phycitodes albatella (Ragonot) [Pyralidae, LE]

Psilus sp. [Diapriidae, HY] do

Reared from Hylemya -...... seneciella (Meade).

Sermylassa halensis (L.) [Chrysomelidae, CO] Reared from plant

Switzerland. France.

Do.

Germany.

France.

Do.

Do.

Do.

Do.

Do.

Do.

Do.

Do.

Do.

Do.

Do.

Do.

Do.

Do.

Do.

Germany.

France.

Germany.

Switzerland

Do.

Do.

Do.

France.

Do.

Do.

Switzerland.

Italy.

France.

Switzerland.

Germany.

Switzerland.

Do.

Italy.

France.

Switzerland.

France.

Do.

See footnotes at end of table. 
Table 2.-Selected weedy plants and associated insects, extent of their association, and country of origin-Continued

Plant and insect ${ }^{1}$

Insect-plant

relationship ${ }^{2}$

Country

Senecio jacobaea L.:-Continued

Sitona lineatus (L.) [Curculionidae, $\mathrm{CO}$ ]

Resting on roots

Sphaerophoria rueppelli Wiedemann [Syrphidae, DI]

Sphenella marginata (Fallén) [Tephritidae, DI]

Reared from head

Trupanea stellata (Fuessly) [Tephritidae, DI] do

do

Trupanea stellata (Fuessly) [Tephritidae, DI]

Trupanea stellata (Fuessly) [Tephritidae, DI] ${ }^{3}$

Tyria jacobaeae (L.) [Arctiidae, LE]

Senecio nemorensis $\mathbf{L}$.:

Cacoecimorpha pronubana (Hübner) [Tortricidae, LE]

Reared from foliage

Senecio squalidus $\mathbf{L}$ :

Peridroma saucia (Hübner) [Noctuidae, LE)

Reared

Silybum marianum (L.) Gaertner:

Apion detritum Rey [Curculionidae, $\mathrm{CO}]$

Reared from stem

Carpocoris mediterraneus Taminini [Pentatomidae, H-HE]

Cassida near deflorata Suffrian [Chrysomelidae, CO]

Cassida prob. vibex L. [Chrysomelidae, CO]

Cassida prob. vibex L. [Chrysomelidae, CO]

Cnepnasia sp. [Tortricidae, LE]

Cynthia cardui (Linnaeus) [Nymphalidae, LE]

Gastroidea polygoni (L.) [Chrysomelidae, CO]

Larinus cynarae (Fabricius) [Curculionidae, CO]

Resting on head -

Feeding on foliage

Larinus cynarae (Fabricius) [Curculionidae, $\mathrm{CO}$ ]

Lixus algirus (L.) [Curculionidae, $\mathrm{CO}]$

Pyroderces argyrogrammos (Zeller) [Cosmopterigidae, LE]

Rhinocyllus conicus (Froelich) [Curculionidae, CO]

---- do

Reared from foliage -------

Reared from head

Reared from foliage

Resting on leaf -

Reared from head

Feeding on head

Resting on stem

Reared from head

do

Tamarix sp.:

Apion sp. [Curculionidae, CO]

Bruchidius near bimaculatus (Olivier) [Bruchidae, CO]

Swept from plant

Bruchidius near imbricornis (Panzer) [Bruchidae, CO]

Bruchidius nudus (Allard) [Bruchidae, CO]

Cantharis sp. [Cantharidae, CO]

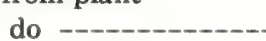

Ceratina sp. [Anthophoridae, HY] -

Chlorophanus excisus (Fabricius) [Curculionidae, $\mathrm{CO}]$

Coccinella septempunctata L. [Coccinellidae, $\mathrm{CO}]$

Coniatus sp. [Curculionidae, $\mathrm{CO}]$

Cteniopus sp. [Alleculidae, $\mathrm{CO}$ ]

Desmometopa near m-nigrum (Zetterstedt) [Milichiidae, DI]

Diorhabda sp. [Chrysomelidae, CO] -

Dolycoris baccarum (L.) [Pentatomidae, H-HE]

Hylemya sp. [Anthomyiidae, DI]

Malachius aeneus L. [Melyridae, CO]

Malachius sp. \#1 [Melyridae, $\mathrm{CO}]$

Malachius sp. \#2 [Melyridae, CO]

do

do - -

do

do

do

do

do

do

do

do

do

do

Microphorus sp. [Empididae, DI]

Minettia plumicornis (Fallén) [Lauxaniidae, DI]

Nabis viridulus Spinola [Nabidae, H-HE] -

Orthellia caesarion (Meigen) [Muscidae, DI]

Pachybrachys sp. [Chrysomelidae, CO]

Platypalpus sp. [Empididae, DI]

Trixoscelis frontalis (Fallén) [Trixoscelidae, DI] -

Trixoscelis obscurella (Fallén) [Trixoscelidae, DI]

Valgus hemipterus L. [Scarabaeidae, CO]

do

do

do

do

do

do

do

do

do

do

do
Italy.

Do.

Italy.

France.

Do.

Germany.

Italy.

France.

Do.

Do.

Do.

Do.

Do.

Do.

Do.

Turkey.

Italy.

Do.

Do.

Do.

Do.

Do.

Turkey.

Do.

Do.

Do.

Do.

Do.

Do.

Do.

Do.

Do.

Do.

Do.

Do.

Do.

Do.

Do.

Do.

Do.

Do.

Do.

Do.

Do.

Do.

Do.

Do.

Do.

See footnotes at end of table. 
Table 2.-Selected weedy plants and associated insects, extent of their association, and country of origin-Continued

Plant and insect ${ }^{1}$

Insect-plant

relationship ${ }^{2}$

Country

Tribulus terestris $\mathbf{L}$.:

Arthrolips sp. [Orthoperidae, CO]

Reared from seed

Italy.

Microlarinus lareynii (Jacq. du Val) [Curculionidae, CO]

Reared from seed pod

Do.

Microlarinus lypriformis (Wollaston) [Curculionidae, CO]

Reared from stem

Do.

Sarcophaga balanina Pandellé [Sarcophagidae, DI]

In soil under plant

Do.

${ }^{1} \mathrm{CO}=$ Coleoptera, $\mathrm{DI}=$ Diptera, H-HE = Hemiptera-Heteroptera, H-HO = Hemiptera-Homoptera, LE $=$ Lepidoptera,

$\mathrm{HY}=$ Hymenoptera, $\mathrm{NE}=$ Neuroptera, OR $=$ Orthoptera.

${ }^{2}$ Dashes indicate no recorded insect-plant relationship.

${ }^{3}$ Host species uncertain, probably Senecio jacobaea L.

\section{INDEX OF INSECT AND PLANT NAMES [Plant names are set in bold type]}

\section{A}

Acalles diocletianus (Germar), 4, 20.

Acalles dromedarius boheman, 4, 17, 20.

Acalles sp., 4, 17.

Acanthiophilus helianthi (Rossi), 8, 15, 16, 17, 18, 20, 24.

Adelphocoris lineolatus (Goeze), 9, 22.

Adelphocoris sp., 10, 22.

Adonia variegata (Goeze), 3, 22

Adscita sp., 14, 19.

Agapanthia cardui (L.), 2, 21.

Agapanthia dahli (Richter), 2, 20, 21.

Agapanthia probably dahli (Richter), 3, 25.

Agrilus biguttatus (Fabricius), 2, 20.

Agromyzidae, 7.

Alleculidae, 2.

Alloeonotus fulvipes (Scopoli), 10.22.

Alophus nictitans Boheman, 4, 25.

Altica carduorum (Guérin-Ménéville), 3, 19.

Anaphes sp., 12, 22.

Anaspis sp. 6, 20.

Ancyrosoma leucogrammum (Gmelin), 10, 25.

Anobiidae. 2.

Anthicidae, 2.

Anthicus sp., 2, 22.

Anthocoridae, 9.

Anthocoris nemorum (L.), 9, 23.

Anthomyiidae, 7.

Anthomyza gracilis Fallén, 7, 16.

Anthomyzidae, 7.

Anthophoridae, 11.

Apanteles sp., 11, 26.

Apion near compactum Desbrochers, 4, 20.

Apion detritum Rey, 4, 15, 19, 27.

Apion near formaneki Wagner, 4, 21.

Apion fuscirostre (Fabricius), 4, 20.

Apion onopordi Kirby, 4, 17, 24.

Apion sp., 4, 17, 19, 21, 23.

Aplocnemus sp., 6, 23.

Arctiidae, 12.
Arctium minus Bernhardi, 15.

Arthrolips sp., 7, 28.

Attalus sp., 6, 17, 18, 22.

\section{B}

Bangasternus sp., 4, 16.

Berytidae, 9.

Berytinus sp., 9, 25.

Biosteres carbonaris (Nees), 11, 20.

Boreava sp., 15.

Brachycoleus decolor Reuter, 10, 23.

Brachypterolus pulicarius (L.), 6, 21, 22, 23.

Brachypterolus sp., 6, 22.

Bracon near osculator Nees, 11, 23.

Bracon sp., 11, 17, 20, 25, 26.

Braconidae, 11.

Bruchidae, 2.

Bruchidius near bimaculatus (Olivier), 2, 27.

Bruchidius near imbricornis (Panzer), 2, 27.

Bruchidius nudus (Allard), 2, 27.

Bruchidius quinqueguttatus (Olivier) complex, 2, 18.

Bruchidius seminarius (L.), 2, 20.

Bruchidius sp. 2, 19.

Bruchidius probably unicolor (Olivier), 2, 22.

Bruchus brachialis Fabricius, 2, 25.

Bruchus dentipes Baudi, 2, 21.

Bufolucilia silvarum (Meigen), 7, 26.

Buprestidae, 2.

\section{C}

Cacoecimorpha pronubana (Hübner), 14, 20, 26, 27.

Caenacis sp., 12, 18.

Callipharidae, 7.

Calophasia casta (Borkhausen), 13, 21, 23.

Camponotus lateralis (Olivier), 12, 23.

Cantharidae, 2.

Cantharis sp., 2, 27.

Cardiophorus near nigerrimus Erichson, 6, 23. 
Carlina corymbosa L., 15.

Carlina lanata L., 15.

Carlina vulgaris L., 15.

Carpocoris lunulatus (Goeze), 10, 23.

Carpocoris mediterraneus Taminini, 10, 23, 27.

Carpocoris pudicus (Poda), 10, 23.

Cartallum ebulinum (L.), 3, 15.

Carthamus lanatus L., 15.

Carthamus tinctorius L., 15.

Cassida near deflorata Suffrian, 3, 27.

Cassida probably vibex L., 3, 19, 27.

Cataglyphis near cursor (Fonscolombe), 12, 23.

Centaurea amara $L_{\text {., }} 15$.

Centaurea caerulescens Willdenow, 15, 16.

Centaurea calcitrapa L., 16.

Centaurea cineraria L., 16.

Centaurea diffusa Lamarck, 16.

Centaurea jacea L., 16.

Centaurea maculosa Lamarck, 17.

Centaurea napifolia L., 17.

Centaurea nicaeensis Allioni, 17.

Centaurea nigra L., 18.

Centaurea paniculata L., 18.

Centaurea phrygia L., 18.

Centaurea repens L., 18.

Centaurea scabiosa L., 18.

Centaurea sp., 18.

Centaurea sphaerocephala L, 18 .

Cerambycidae, 2, 3.

Ceratina sp., 11, 27.

Cercopidae, 10.

Cercopis vulnerata Rossi, 10, 23.

Ceutorhynchidius horridus (Panzer), 4, 21.

Ceutorhynchus litura (Fabricius), 4, 19.

Ceutorhynchus trimaculatus (Fabricius), 4, 21.

Chaetocnema probably conducta Motschulsky, 3, 25.

Chaetorellia sp., 8, 15, 16.

Chaetostomella onotrophes (Loew), 8, 16, 18, 19.

Chamaemyiidae, 7.

Chamaesphecia empiformis (Esper), 14, 21.

Chelonus sp., 11, 22.

Chlorissa sp., 13, 25.

Chlorophanus excisus (Fabricius), 4, 27.

Chloropidae, 7.

Chrysolina grossa (Fabricius), 3, 25.

Chrysolina gypsophilae Küster, 3, 21.

Chrysolina hyperici Forster, 3, 21.

Chrysolina sp., 3, 22, 23.

Chrysolina variolosa Pethena, 2, 23.

Chrysomela sp. or near, 3, 20.

Chrysomelidae, 3.

Chrysopa near carnea Stephens, 14, 23.

Chrysopidae, 14.

Cicadatra near hyalina (Fabricius), 10, 23.

Cicadellidae, 10.

Cicadidae, 10.

Circulifer opacipennis (Lethierry), 10, 18.

Cirsium arvense (L.) Scopoli, 19.

Cirsium odontolepis Boissier, 19.

Cirsium sp., 20.

Cirsium vulgare (Savi) Tenore, 19, 20.

Cixiidae, 10.
Cleonus morbillosus (Fabricius), 4, 19.

Cleonus piger (Scopoli), 4, 15, 19, 24.

Clytra atraphaxidis Pallas or near, 3, 18.

Clytra laeviuscula Ratzeburg or near, 3, 23.

Cnephasia sp., 14, 17, 27.

Coccinella quinquepunctata L., 4, 23, 27.

Coccinella septempunctata L., 4, 23.

Coccinellidae, $3,4$.

Cochylidae, 12.

Cochylis probably atricapitana (Stephens), 12, 25, 26.

Cochylis near hybridella (Hübner), 12, 19.

Cochylis posterana (Zeller), 12, 16.

Coleophora sp., 13, 17, 25.

Coleophoridae, 12.

Colotes sp., 6, 23.

Coniatus sp., 4, 27.

Probably Coptocephala sp., 3, 18.

Coreidae, 9.

Corizus hyoscyami L., 10, 23.

Corticaria sp., 6, 17, 19.

Cosmopterigidae, 12, 13.

Crepidodera ferruginea (Scopoli) or near, 3, 24.

Cryptocephalus hypochoeridis (L.), 3, 24.

Cryptocephalus populi Suffrian or near, 3, 25.

Cteniopus sp. 2, 27.

Curculionidae, 4, 5, 6.

Cymus glandicolor Hahn, 9, 22.

Cynara cardunculus L., 20.

Cynara scolymus $L_{\text {. }} 20$.

Cynipidae, 11.

Cynthia cardui (L.), 13, 25, 27.

Cytisus scoparius (L.) Link, 20.

Cytisus sp., 20.

\section{D}

Dacnusa leptogaster Haliday, 11, 22.

Danacaea sp., 6, 16.

Probably Danacaea sp., 6, 22, 23.

Deltocephalus sp., 10, 18.

Depressaria sp., 13, 16.

Deraeocoris schach (Fabricius), 10, 24.

Desmometopa near m-nigrum (Zetterstedt), 8, 27.

Diapriidae, 11.

Dictyla echii (Schrank), 10, 23.

Diglyphosema sp., 11, 26.

Diorhabda sp., 3, 27.

Divales bipustulata Fabricius, 6, 15.

Divales sp., 6, 15.

Dolycoris baccarum (L.), 10, 23, 24, 27.

Drosophila funebris (Fabricius), 7, 20.

Drosophilidae, 7.

\section{E}

Echinops sphaerocephalus L., 21.

Elateridae, 6.

Ematurga atomaria (L.), 13, 26.

Empididae, 7.

Enoplops discigera (Kolenati), 9, 23.

Entedon sp., 11, 23.

Epiblema near pflugiana (Fabricius), 14, 19.

Epuraea sp., 6, 19. 
Eucosma campoliliana (Denis \& Schiffermüller), 14, 26.

Eudecatoma biguttata Swederus or E. submutica (Thomson), $11,15,16,17$.

Eudecatoma submutica (Thomson), 11, 15, 16, 17.

Eulophidae, 11.

Eumerus sp., 8, 26.

Eumerus strigatus Fallén, 8, 18.

Eupelmidae, 11.

Eupelmus sp., 11, 23.

Euphorbia esula L., 21.

Euphorbia sp., 21.

Eupithecia absinthiata (Clerck), 13, 26.

Eupithecia centaurearia (Denis \& Schiffermüller), 13, 25.

Eupithecia linariata (Denis \& Schiffermüller), 13, 22.

Eupithecia sp., 13, 19.

Eurydema ornatum L., 10, 21.

Eurygaster maura (L.), 10, 23.

Eurytoma robusta Mayr, 12, 15, 16, 17, 18, 19.

Eurytoma robusta Mayr or E. tibialis Boheman, 12, 21, 24.

Eurytoma sp., 12, 23.

Eurytoma tibialis Boheman, 12, 15, 16, 17, 18, 19.

Eurytomidae, 11, 12.

Eutrias sp., 11, 26.

Exartema sp., 14, 26.

Eysarcoris inconspicuus (Herrich-Schäffer), 10, 23.

\section{$\mathbf{F}$}

Fannia sp., 8, 26.

Formica near nasuta Nylander, 12, 23.

Formica sp., 12, 23.

Formicidae, 12.

\section{G}

Galactites tomentosa Moench, 21.

Gastroidea polygoni (L.), 3, 20, 25, 27.

Genista sagittalis L., 21.

Genista sp., 21.

Geocoris megacephalus siculus (Fieber), 9, 25.

Geometridae, 13.

Glaphyrus sp., 7, 24.

Graphosoma lineata (L.), 10, 24.

Gymnancyla canella (Denis \& Schiffermüller), 14, 25.

Gymnetron antirrhini (Paykull), 4, 22.

Gymnetron linariae (Panzer), 4, 22.

Gymnetron netum (Germar), 4, 22.

Gymnetron sp., 4, 17, 21, 22.

Gymnoscelis pumilata (Hübner), 13, 22.

\section{$\mathbf{H}$}

Handianus sp., 10, 18.

Heliothis peltiger (Denis \& Schiffermüller), 13, 16.

Heliothis sp., 13, 23.

Hephathus nanus (Herrich-Schäffer), 10, 13.

Heterogaster urticae (Fabricius), 9, 25.

Holcostethus sp., 10, 18.

Holcostethus strictus (Fabricius), 10, 22.

Homeosoma near nebulella (Denis \& Schiffermüller), 13, 19.
Homeosoma sp., 13, 20.

Hyalesthes obsoletus Signoret, 11, 18.

Hylemya brassicae (Wiedemann), 7, 26.

Hylemya cinerella (Fallén), 7, 26.

Hylemya coarctata (Fallén), 7, 26.

Hylemya platura (Meigen), 7, 26.

Hylemya pullula (Zetterstedt), 7, 26.

Hylemya radicum (L.), 7, 15, 26.

Hylemya seneciella (Meade), 7, 26.

Hylemya sp., 7, 27.

Hyles euphorbiae (L.), 14, 21.

Hypera postica (Gyllenhal), 4, 17.

Hypericum sp., 21.

Hysteropterum grylloides (Fabricius), 11, 13.

\section{I}

Isocolus scabiosae (Giraud), 11, 15, 17, 18. Issidae, 10.

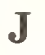

Julistus sp., 6, 2?

\section{$\mathbf{L}$}

Larinus australis Capiomont, 5, 18, 24.

Larinus probably australis Capiomont, 5, 18.

Larinus cynarae (Fabricius), 5, 24, 27.

Larinus flavescens Germar, 5, 15, 16, 17.

Larinus jaceae (Fabricius), 5, 15.

Larinus latus (Herbst), 5, 24.

Larinus near leuzeae Fabre, 5, 18.

Larinus longirostris Gyllenhal, 5, 16, 17.

Larinus planus (Fabricius), 5, 15, 16, 19.

Larinus rusticanus Gyllenhal, 5, 15.

Larinus scolymi (Olivier), 5, 20.

Larinus sturnus (Schaller), 5, 16, 19.

Larinus turbinatus Gyllenhal, 5, 20.

Larinus ursus (Fabricius), 5, 15.

Lasioderma baudii (Schilsky), 2, 20, 26.

Lasioderma probably obscurum Solsky, 2, 15.

Lasioderma serricorne (Fabricius), 2, 16, 17.

Lathridiidae, 6.

Lauxaniidae, 7

Lema cyanella L., 3, 19.

Leptocera sp., 8, 21.

Leucocelis sp., 7, 23.

Leucopis sp., 7, 23.

Leucoptera spartifoliella (Hübner), 13, 20.

Linaria dalmatica (L.) Miller, 21.

Linaria fastigiata Chavannes, 21.

Linaria genistifolia (L.) Miller, 21.

Linaria grandiflora Desfontaines, 21.

Linaria heterophylla Desfontaines, 22.

Linaria kurdica Boissier \& Hohenacker, 22.

Linaria lineolata Boissier, 22.

Linaria repens (L.) Miller, 22.

Linaria sp., 22, 23, 24.

Linaria vulgaris Miller, 22. 
Liriomyza latigenis (Hendel), 7, 26.

Liriomyza strigata (Meigen), 7, 25.

Lixus algirus (L.), 5, 20, 27.

Lixus cardui Olivier, 5, 24.

Lixus elongatus (Goeze), 5, 20.

Lixus punctiventris Boheman, 5, 25, 26.

Lonchoptera furcata (Fallén), 8, 17.

Lonchopteridae, 7.

Longitarsus dorsalis (Fabricius), 3, 25.

Longitarsus ganglbaueri Heikertinger, 3, 25.

Longitarsus probably ganglbaueri Heikertinger, 3, 25, 26.

Longitarsus jacobaea Waterhouse, 3, 25.

Longitarsus probably jacobaea Waterhouse, 3, 25, 26.

Longitarsus sp., 3, 26.

Longitarsus near tabidus (Fabricius), 3, 25.

Lygaeidae, 9.

Lygaeus equestris (L.), 9, 23.

Lygus pratensis (L.), 10, 23.

Lygus rugulipennis Poppius, 10, 23.

Lyonetiidae, 13.

\section{M}

Malachius aeneus L. 6, 27.

Malachius sp., 6, 27.

Mecinus janthinus (Germar), 5, 22.

Mecinus sp., 5, 22, 23.

Melanagromyza aeneoventris (Fallén), 7, 26.

Melanagromyza dettmeri Hering, 7, 25.

Melanagromyza eupatorii Spencer, 7, 26.

Melanagromyza near tripolii Spencer, 7, 25.

Meligethes incanus Sturm, 6, 17, 25.

Meligethes sp., 6, 19.

Melitaea cinxia (L.), 13, 20.

Melitaea didyma (Esper), 13, 22, 23.

Meloidae, 6.

Melyridae, 6.

Mesocerus marginatus (L.)?, 9, 24, 25.

Microlarinus lareynii (Jacq. du Val), 5, 28.

Microlarinus lypriformis (Wollaston), 5, 28.

Microphorus sp., 7, 27.

Microplitis possibly scrophulariae Szelenyi, 11, 23.

Microplitis possibly varitpes Ruthe, 11, 23.

Milichiidae, 8.

Minettia plumicornis (Fallén), 8, 27.

Miridae, 9, 10.

Mordellidae, 6.

Mordellistena sp., 6, 17.

Muscidae, 8.

Myelois cribrella (Hübner), 13, 15.

Myelois sp., 13, 24.

Mylabris apicipennis Reiche, 6, 23.

Mylabris sp., 6, 23.

Mymaridae, 12.

\section{$\mathbf{N}$}

Nabidae, 10.

Nabis viridulus Spinola, 10, 27.

Nemotelus sp., 8, 23.

Nezara viridula (L.), 10, 21.
Nitidulidae, 6.

Noctuidae, 13.

Nymphalidae, 13.

\section{O}

Oecophoridae, 13.

Oedemera podagrariae (L.), 6, 24.

Oedemeridae, 6.

Oliarus barajus Diabola, 11, 18.

Olibrus corticalis (Panzer), 7, 26.

Omophulus sp., 2, 24.

Onopordum acanthium L., 24.

Onopordum sp., 24.

Onopordum tauricum Willdenow, 24.

Ophiomyia senecionina Hering, 7, 26.

Opius sp., 11, 26.

Orellia colon (Meigen), 8, 15.

Orellia near colon (Meigen), 8, 18.

Orellia falcata (Scopoli), 8, 16.

Orellia lappae (Cederhjelm), 9, 24.

Orellia punctata (Schrank), 9, 17.

Orellia near punctata (Schrank), 9, 17.

Orellia sp., 9, 18.

Orgilus sp., 11, 26.

Ormyridae, 12.

Ormyrus gratiosus (Foerster), 12, 16.

Ormyrus punctiger Westwood, 12, 15, 16, 17, 19, 24.

Orthellia caesarion (Meigen), 8, 27.

Orthoperidae, 6.

Oscinella frit (L.), 7, 16.

Oscinella sp., 7, 21.

Otiorhynchus ovatus (L.), 5, 17.

Otiorhynchus rugostriatus (Goeze), 5, 26.

Otiorhynchus sp., 5, 19.

Otitidae, 8.

Oulema melanopus (L.), 3, 26.

Oxyna nebulosa (Wiedemann), 9, 26.

\section{$\mathbf{P}$}

Pachybrachys sp., 3, 27.

Palomena prasina (L.), 10, 24.

Palpita unionalis (Hübner), 13, 22.

Pantomorus cervinus (Boheman), 5, 26.

Paragus sp., 8, 24.

Parmena sp., 3, 16.

Paroxyna probably tessellata (Loew), 9, 26.

Pentatomidae, 10.

Peridroma saucia (Huibner), 13, 27.

Phalacrididae, 6.

Philaenus spumarius (L.), 10, 19, 24, 26.

Phloeotribus rhododactylus (Marsham), 7, 20.

Phora sp., 8, 19.

Phoridae, 8.

Phrydiuchus spilmani Warner, 5, 25.

Phrydiuchus tau Warner, 5, 25.

Probably Phycitodes albatella (Ragonot), 13, 26.

Phycitodes carlinella (Heinemann), 13, 26.

Phyllobius sp., 5, 20.

Phyllotreta sp., 3, 15.

Phymata crassipes (Fabricius)?, 10, 25. 
Phymatidae, 10.

Phytodecta oblivacea Forster, 3, 20.

Phytoecia pustulata (Schrank), 3, 25.

Phytoecia sp., 3, 21, 22.

Phytometra sp., 13, 24, 25.

Phytomyza probably horticola Goureau, 7, 16, 24.

Platypalpus sp., 7, 21, 27.

Plutella xylostella (L.), 13, 24.

Plutelltidae, 13.

Podabrus sp., 2, 21.

Podonta aubei Mulsant, 2, 24.

Podopidae, 10.

Polymerus sp., 10, 24.

Polyodaspis ruficornis (Macquart), 7, 16.

Porphyrinia parva (Hübner), 13, 15, 16.

Porphyrinia purpurina (Denis \& Schiffermüller), 13, 19.

Prodotis stolida (Fabricius), 13, 15, 20.

Psilothrix sp., 6, 18.

Psilus sp., 11, 26.

Pteromalidae, 12.

Ptinidae, 7.

Ptinus villiger (Reitter), 7, 16.

Pyralidae, 13.

Pyroderces argyrogrammos (Zeller), 13, 15, 16, 18, 19, 20 , $21,24,27$.

\section{Q}

Quercus pubescens Willdenow:, 25.

\section{$\mathbf{R}$}

Reduviidae, 10.

Rhinocoris iracundus Poda, 10, 24.

Rhinocyllus conicus (Froelich), 5, 16, 18, 27.

Rhopalidae, 10.

Rhopalus sp., 10, 24.

Rhyzobius litura (Fabricius), 4, 17.

\section{$\mathbf{S}$}

Salsola kali L., 25.

Salsola sp., 25.

Salvia aethiopis L., 25.

Salvia argentea, L., 25.

Salvia limbata C.A. von Meyer, 25.

Salvia palaestina Bentham, 25.

Salvia sp.:, 25.

Salvia verbenaca $L$., 25.

Salvia virgata Jacquin, 25.

Sarcophaga balanina Pandelle, 8, 28.

Sarcophaga sp., 8, 16.

Sarcophagidae, 8.

Scarabaeidae, 7 .

Scelionidae, 12.

Scolytidae, 7.

Scrophularia canina L., 25.

Scymnus sp., 4, 15, 24.

Scymnus (s. str.) sp., 4, 24.
Senecio aquaticus Hill, 25, 26.

Senecio ercifolius L., 26.

Senecio jacobaea L., 26, 27.

Senecio nemorensis L., 27.

Senecio squalidus L., 27.

Sermylassa halensis (L.), 3, 27.

Sesiidae, 14.

Sibinia attalica (Gyllenhal), 5, 15, 16.

Silybum marianum (L.), Gaertner, 27.

Sitona gressorius (Fabricius), 5, 20.

Sitona lineatus (L.), 5, 27.

Sitona puncticollis Stephens, 5, 26.

Sitona sp., 6, 16, 19, 20, 21, 24.

Sitophilus sp., 6, 20.

Spermophagus sericeus (Geoffroy), 2, 18, 25.

Sphaeroceridae, 8.

Sphaeroderma rubidum (Graells), 3, 19, 20.

Sphaeroderma testaceum L., 3, 19.

Sphaerophoria rueppelli Wiedemann, 8, 27.

Sphenella marginata (Fallén), 9, 27.

Sphingidae, 4.

Sphragisticus nebulosus (Fallén), 9, 25.

Spilostethus saxatilis (Scopoli), 9, 24.

Spintherus sp., 12, 24.

Stagmatophora serratella (Treitschke), 13, 21, 22, 24.

Stictopleurus punctatonervosus (Coeze), 10, 19.

Stratiomyidae, 8.

Strobilotoma typhaecornis (Fabricius), 9, 25.

Strophomorphus porcellus (Schonherr), 6, 21.

Subcoccinella virgintiquattuorpunctata (L.), 4, 20, 24.

Syrphidae, 8.

\section{$\mathbf{T}$}

Tachinidae, 8.

Tamarix sp., 27.

Tapinoma sp., 12, 24.

Telenomus sp., 12, 24.

Tephritidae, 8.

Tephritis postica (Loew), 9, 24.

Terellia fuscicornis (Loew), 9, 20.

Terellia longicauda (Meigen), 9, 20.

Terellia serratulae (L.), 9, 18.

Terellia virens (Loew), 9, 17.

Terellia near virens (Loew), 9, 24.

Tettigometra griseola Fieber, 11, 18.

Tettigometra sp., 11, 18.

Tettigometridae, 11.

Tettigonia caudata (Charpentier), 14, 24.

Tettigoniidae, 14.

Thaumatomxia sulcifrons (Becker), 7, 18.

Timia xanthaspis (Loew), 8, 18.

Tingidae, 10.

Tortricidae, 14.

Tribulus terrestris $\mathbf{L}_{\text {., }} 28$.

Trixoscelidae, 9.

Trixoscelis frontalis (Fallén), 9, 27.

Trixoscelis obscurella (Fallén), 9, 27.

Trupanea stellata (Fuessly), 9, 27.

Tychius quinquepunctatus (L.), 6, 19.

Tyria jacobaeae (L.), 12, 27. 
U

Urophora affinis (Frauenfeld), 9, 17.

Urophora cuspidata (Meigen), 9, 18.

Urophora jaceana (Hering), 9, 18.

Urophora quadrifasciata (Meigen), 9, 16, 17.

Urophora probably quadrifasciata (Meigen), 9, 15, 16, 17, 18.

Urophora stylata (Fabricius), 9, 19, 20.
V

Valgus hemipterus L., 7, 27.

Voria ruralis (Fallén), 8, 20.

$\mathbb{Z}$

Zygaenidae, 14. 


U.S. DEPARTMENT OF AGRICULTURE SCIENCE AND EDUCATION ADMINISTRATION WASHINGTON, D.C. 20250

OFFICIAL BUSINESS

PENALTY FOR PRIVATE USE, \$300
POSTACE AND FEES PAID

U. S. DEPARTMENT OF AGRICULTURE

ACR 101

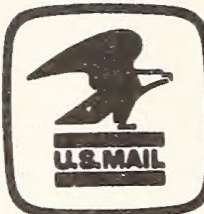

